\title{
Cretaceous magmatic evolution in the Deylaman igneous complex, Alborz zone, Iran: change from extensional to compressional regime
}

\author{
Sheida AKMALI ${ }^{1}$, Abbas ASIABANHA ${ }^{1, *}$ and Shahrooz HAGHNAZAR ${ }^{2}$ \\ 1 Department of Geology, Faculty of Science, Imam Khomeini International University, Qazvin 34148-96818, Iran \\ 2 Department of Geology, Islamic Azad University, Lahijan Branch, Lahijan, Iran
}

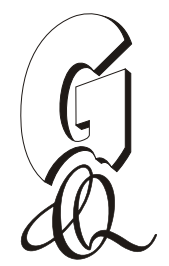

Akmali, S., Asiabanha, A., Haghnazar, S., 2019. Cretaceous magmatic evolution in the Deylaman igneous complex, Alborz zone, Iran: change from extensional to compressional regime. Geological Quarterly, 63 (4): 757-770, doi: 10.7306/gq.1500

Associate editor: Tomasz Bajda

\begin{abstract}
The Deylaman igneous complex, as a part of the Late Cretaceous rock unit that lies behind the Paleogene Alborz magmatic arc, in the northern Alborz zone, is composed of basaltic sheet lavas alternating with the pelagic calcareous sediments, basaltic pillow lavas, felsic lavas and gabbroic-monzodioritic intrusions. The pelagic calcareous deposits contain microfossils representing the Santonian-Maastrichtian ages. Furthermore, petrographic textures such as the hyalomicrolitic texture and swallow-tail plagioclase crystals in the pillow lavas, and also segregation vesicles in the basaltic sheet lavas, imply high external (hydrostatic) pressures as the magma was extruded in a deep-water environment. The rock samples show both compositional bimodality and characteristic trends in the variation diagrams. Also, some geochemical characteristics imply that the basaltic lavas originated from the partial melting of an undepleted deep mantle source containing spinel Iherzolite: the enrichment patterns of LREE/HREE ratios of the samples $[(\mathrm{La} / \mathrm{Yb}) \mathrm{n}=3.93-4.16$ for basaltic lavas and 10.92 for felsic lavas] lying between those characteristic of OIBs $[(\mathrm{La} / \mathrm{Yb}) \mathrm{n}=12.92]$ and EMORBs $[(\mathrm{La} / \mathrm{Yb}) \mathrm{n}=1.91]$; similarities between the patterns of multi-element spider-diagrams; LILE bulges in the basaltic samples compared with those of OIBs. Moreover, the samples show influence from two geotectonic environments: supra-subduction zone (SSZ) settings and plume-type within-plate magmas. Therefore, because of the deep submarine environment inferred for the effusive volcanic eruptions in Santonian-Maastrichtian time, it seems that the Deylaman igneous complex evolved through two stages: first, a tensional regime in a supra-subduction zone (farther from the Mesozoic magmatic arc) and formation of an embryonic rift-related oceanic basin in the Late Jurassic-Early Cretaceous; secondly, a compressive regime in the Late Cretaceous-Early Paleocene and inland migration of the magmatic arc. Consequently, the Cretaceous magmatism can be interpreted as a prelude to the Eocene magmatic flare-up in the magmatic arcs of Iran.
\end{abstract}

Key words: back-arc magmatism, Cretaceous, Neotethys, Alborz, Iran.

\section{INTRODUCTION}

The Cretaceous magmatic events in Iran [e.g., the Cretaceous ophiolitic sequences and also the Cretaceous arc volcanism in the different areas such as the Sanandaj-Sirjan magmatic arc (SSMA), the Urumieh-Dokhtar magmatic arc (UDMA), the Alborz and Makran zones] are closely related to the tectonic events of the Tethyan realm, which began in the Early Jurassic as a result of subduction of the Neotethyan oceanic lithosphere below the southern margin of the Eurasia supercontinent (Berberian and King, 1981) and continued throughout the Cretaceous (Stampfli and Borel, 2004). The event is reflected by the ophiolite sequences of the Zagros

\footnotetext{
* Corresponding author, e-mail: asiabanha@sci.ikiu.ac.ir

Received: October 19, 2018; accepted: September 9, 2019; first published online: December 16, 2019
}

orogeny and by volcanic-plutonic activities in the SSMA. During this time, the oceans around the central Iranian microcontinents were closed and the bordering ophiolites and coloured mélanges (e.g., Esfandagheh, Nain-Baft and Sabzevar) were emplaced (e.g., Stampfli and Borel, 2004).

At the same time, volcanic events occurred in the inner parts of Iran, such as in the Urumieh-Dokhtar magmatic arc (UDMA) and the Alborz zone (e.g., Taki, 2017; Jafari Sough et al., 2018; Yadollah-Pour et al., 2019); but they are more sporadic and less intense than in the SSMA and possibly were formed in a different environment. Because of this, many authors (e.g., Agard et al., 2011; Chiu et al., 2013) proposed magmatic quiescence in the UDMA in the Cretaceous. Agard et al. (2011) suggest that this volcanic cessation is broadly coincident with the temporal shift of magmatism from the supra-subduction zone (SSZ) to the UDMA and with a slowing of convergence velocities. Hassanzadeh and Wernicke (2016) suggest a slab flattening event in the Cretaceous for the inland migration of the magmatic arc.

In the Alborz zone, Cretaceous volcanic rocks are seen in two stratigraphic situations: (1) as massive basaltic lava flows 
underlain by the Early Cretaceous Tizkuk Formation (Annells et al., 1975; Taki, 2017; Jafari Sough et al., 2018); (2) as intercalations within the carbonate strata of the Cretaceous Chalus Formation (Yadollah-Pour et al., 2019). Moreover, Lam (2002) reported the $98 \mathrm{Ma}$ Nusha pluton in the central Alborz zone, dated by the ${ }^{40} \mathrm{Ar} /{ }^{39} \mathrm{Ar}$ method.

The Deylaman igneous complex (DIC) located behind the Eocene Alborz magmatic arc (Fig. 1) is one of the few Cretaceous igneous outcrops in the Alborz zone. The DIC is important in the sense that it can be assumed as a prelude to the Cenozoic magmatic flare-up. Therefore, the description and discussion of their stratigraphy and petrology can clarify some ambiguities; especially: (1) what was the eruption environment and tectonic setting of the Cretaceous magmatism in the area studied?; (2) what are the relations between the DIC and other concurrent events in the nearby zones (i.e. those of the SSMA and UDMA)? (3) is there any relationship between Mesozoic and the Cenozoic volcanism in the area? For this study, we focus on the Deylaman area.

\section{GEOLOGICAL SETTING}

A relatively deep sedimentary basin has been proposed for the northern slopes of the Alborz zone as indicated by the greater thickness of the Paleozoic and Mesozoic deposits in the northern Alborz relative to the southern Alborz (Alavi, 1996). According to Alavi (1996), the Cretaceous rocks in the Alborz zone constitute a sequence of Orbitolina-bearing limestones associated with intraformational conglomerate, basaltic lavas and diabasic sills. He suggests that these sedimentary characteristics reflect tectonic instabilities in the basin and also transgression of a shallow-marine, epicontinental/continental shelf environment onto Lower Jurassic foreland deposits during Middle Jurassic time. Such a basin in the western and central Alborz began to be disrupted in the Late Cretaceous, and a distinctive magmatic activity was initiated that was followed by intense arc-type magmatism, thrust faulting, folding, uplift and formation of intermontane siliciclastic basins. On the other hand, Berberian and King (1981) suggested that the Jurassic and Cretaceous volcanic products of the Caucasus and northwestern Iran was the result of closing of the Sevan-Akera Ocean during the Late Neocomian-Albian (118-105 Ma) when NW Iran collided with the Pontian-Transcaucasus island arc.

The DIC (Fig. 1), is situated in the northern Alborz zone and its Cretaceous succession can be divided into following units (Fig. 2):

Basaltic lavas. - The basaltic rocks of the area are grouped into two types (Fig. 3): (1) sheet lavas alternating with pelagic carbonates (Fig. 3A). The latter deposits contain microfossils representing the Santonian-Maastrichtian ages and an open, deep marine environment (Hultberg and Malmgren, 1986; Shahin, 1992; Keller et al., 2002; Luciani, 2002). The thickness of each lava sheet is $\sim 2-3 \mathrm{~m}$ and they are grey-black in colour; (2) the pillow lavas (Fig. 3B), 0.5-1 m or more in diameter, are circular and undeformed in shape and have cracks especially in their margins. Although no clear field relations have been observed between the two types of lava, it seems that the pillow lavas are stratigraphically overlain by the sheet lavas.

Felsic lavas. - The basaltic sheet lavas in the area are sporadically overlain or cut by small lenticular and or dyke-like bodies (Fig. 3C) of felsic rocks (trachyte or alkali rhyolite in composition).
Intrusive bodies. - In the DIC, the gabbroic (Fig. 3D) and monzodioritic rocks are seen as grey-black and small (about 1-2 km across) bodies with the same chemical and mineralogical composition as the basaltic rocks.

\section{ANALYTICAL METHODS}

In order to determine the petrographic and geochemical characteristics of the lithological sequence of the area, the freshest samples were taken during field surveying ( 50 samples), among which 10 of the freshest samples were selected for whole-rock chemical analysis. The samples were analyzed at the geochemistry laboratory of the Geological Society of Iran (GSI) using X-ray fluorescence (XRF) for major element oxides and inductively coupled plasma mass spectrometry (ICP-MS) for trace elements. Samples were crushed and pulverized to 60-70 $\mu \mathrm{m}$. Major elements were determined after preparation as pressed pellets containing $4 \mathrm{~g}$ of each sample and $0.8 \mathrm{~g}$ Hoechst Wax-C after homogenization in an agate mortar. For trace and rare earth elements, $0.5 \mathrm{~g}$ of each sample was mixed with $10 \mathrm{ml}$ of hydrofluoric acid and $3 \mathrm{ml}$ perchloric acid and finally was heated to $160^{\circ} \mathrm{C}$.

To achieve more comprehensive results and interpretations, we have also used published data (Haghnazar and Malakotian, 2009; Haghnazar, 2012; Haghnazar et al., 2015, 2016) on similar outcrops (i.e. the Javaher-Dasht and Spilli districts in the eastern Guilan province, N Iran) in the surrounding area. Representative results of these other sources and the results of this study (shown by a star symbol) are shown in Table 1.

\section{PETROGRAPHY}

Basaltic lavas. - The pillow lavas are very fine-grained $(0.05-0.15 \mathrm{~mm}$ in diameter) with hyalo-aphanitic texture and show plagioclase (60 vol\%), olivine (8 vol\%), clinopyroxene (4 vol\%) and opaque minerals (3 vol\%) set in a hyalomicrolitic matrix (Fig. 4A). The sheet lavas are of similar mineralogical composition to the pillow lavas but with coarser-grained textures (with the 2-3 $\mathrm{mm}$ sized microphenocrysts in a hyalo-microlithic matrix; Fig. 4B). Euhedral-subhedral phenocrysts and occasional rounded to corroded phenocrysts of clinopyroxene as well as segregation vesicles (Fig. 4C) are the outstanding petrographic features of these samples.

Felsic lavas. - The felsic rocks include trachytes and alkali rhyolites that are characterized by phenocrysts of sanidine (50-70 vol\%), plagioclase (5-10 vol\%), pyroxene ( 5 vol\%), quartz (5-30 vol\%) and opaque minerals with a hyalo-porphyritic texture (Fig. 4D). Also, small amounts of olivine ( 3 vol\%), either poikilitically in the alkali feldspars or as separate phases, and also apatite ( $1 \mathrm{vol} \%)$ are seen in the trachytes and alkali rhyolites. Furthermore, minor poikilitic, sinusoidal, glomeroporphyritic and felsitic textures are seen.

Intrusive rocks. - Olivine gabbros and monzodiorites comprise the intrusive bodies in the study area. The olivine gabbros contain plagioclase (45 vol\%), olivine (20 vol\%), clinopyroxene (18 vol\%), apatite ( 2 vol\%) and opaque minerals (15 vol\%) with an intergranular texture. The monzodiorites are characterized by plagioclase (70 vol\%), alkali feldspar (20 vol\%), clinopyroxene (6 vol\%), apatite ( $1 \mathrm{vol} \%$ ) and opaque minerals (3 vol\%) set in a granular texture. 


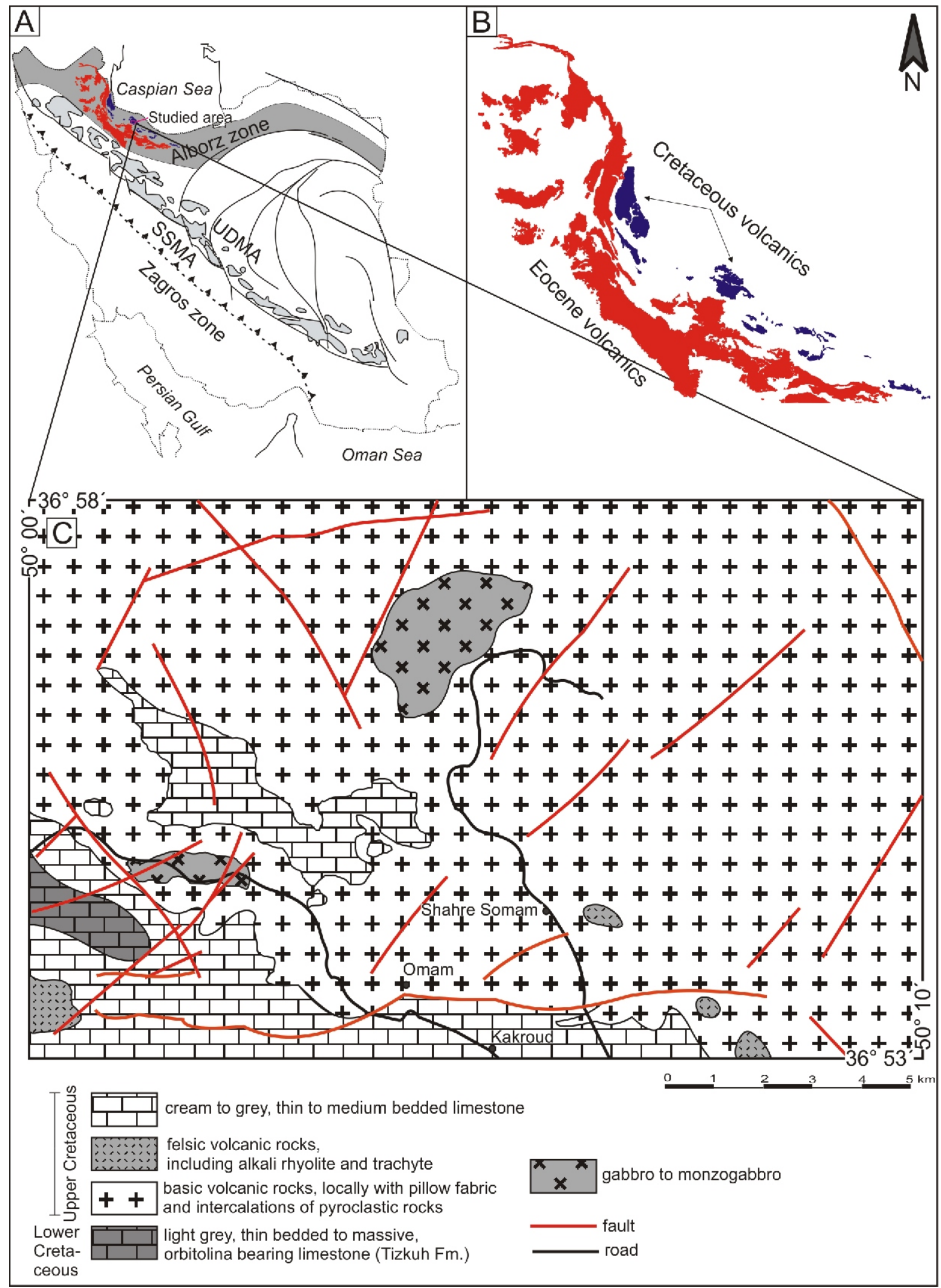

Fig. 1A, B - the location of the Deylaman district in the structural map of Iran; C - geological map of the Deylaman igneous complex (DIC) modified after Baharfiruzi et al. (2003) 


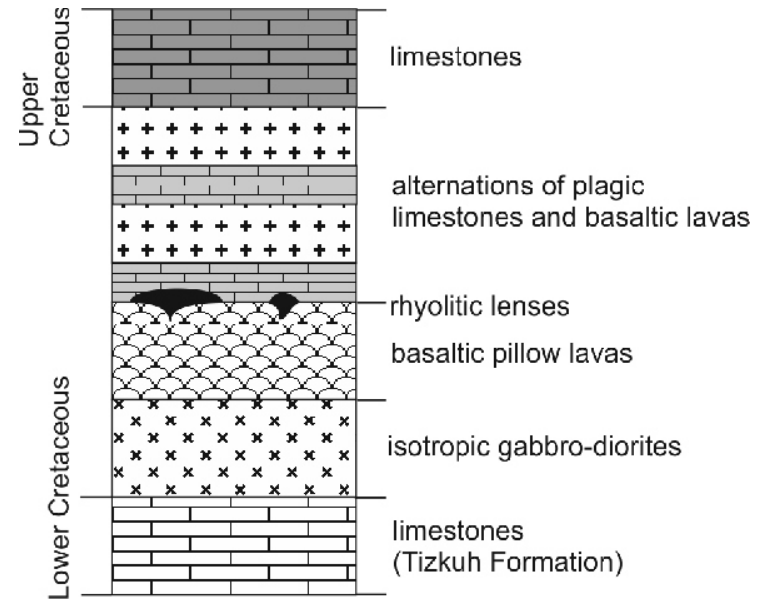

Fig. 2. A stratigraphic column of the geological units in the Deylamn district, Iran
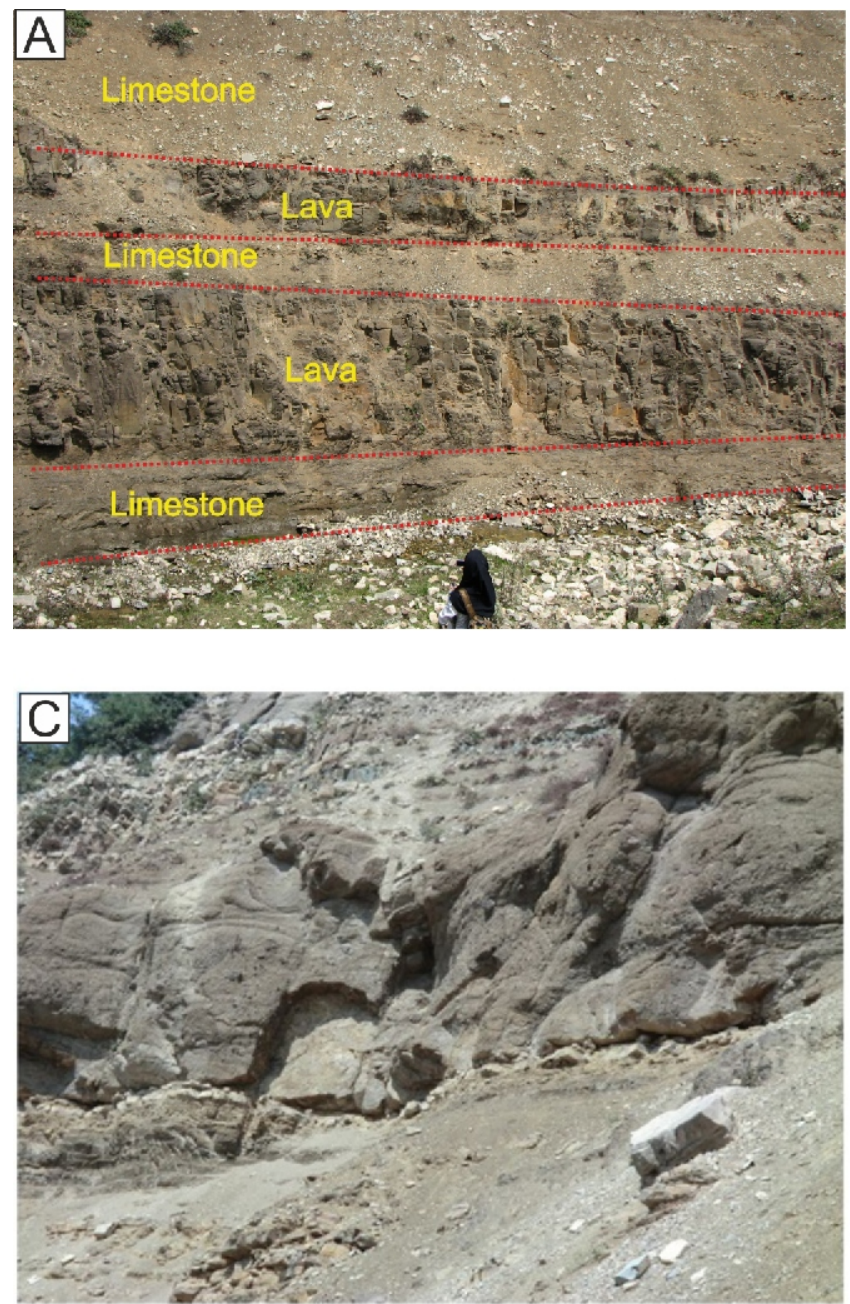

\section{GEOCHEMISTRY}

As shown in Table 1, there is a wide variety of chemical compositions among the samples; for example: $\mathrm{SiO}_{2}=$ 45.13-73.7 wt. \%; $\mathrm{MgO}=0.03-10.81$ wt. $\% ; \quad \mathrm{CaO}=$ 0.09-13.59 wt. \%; $\mathrm{Al}_{2} \mathrm{O}_{3}=11.9-18.29$ wt. \%. Figure $5 \mathrm{~A}$ shows the compositional distribution of the samples on a TAS diagram. Because of the effects of post-solidification alteration and of prolonged weathering, especially in the relatively ancient rocks, the samples show very variable amounts of L.O.I., between 0.45 to 3.86 wt. \% (Table 1). Therefore, we use the immobile elements for their classification, such as the diagram of Winchester and Floyd (1977) modified by Pearce (1996). In this diagram (Fig. 5B), the pillow lavas and intrusive bodies fall in the alkali basalt field, while the sheet lavas set within basaltic andesites and the felsic lavas belong to the trachytes-alkali rhyolites. The samples also show a compositional bimodality in the plot of Winchester and Floyd (1977) and also in the TAS diagram of Le Maitre et al. (2002; Fig. 5).
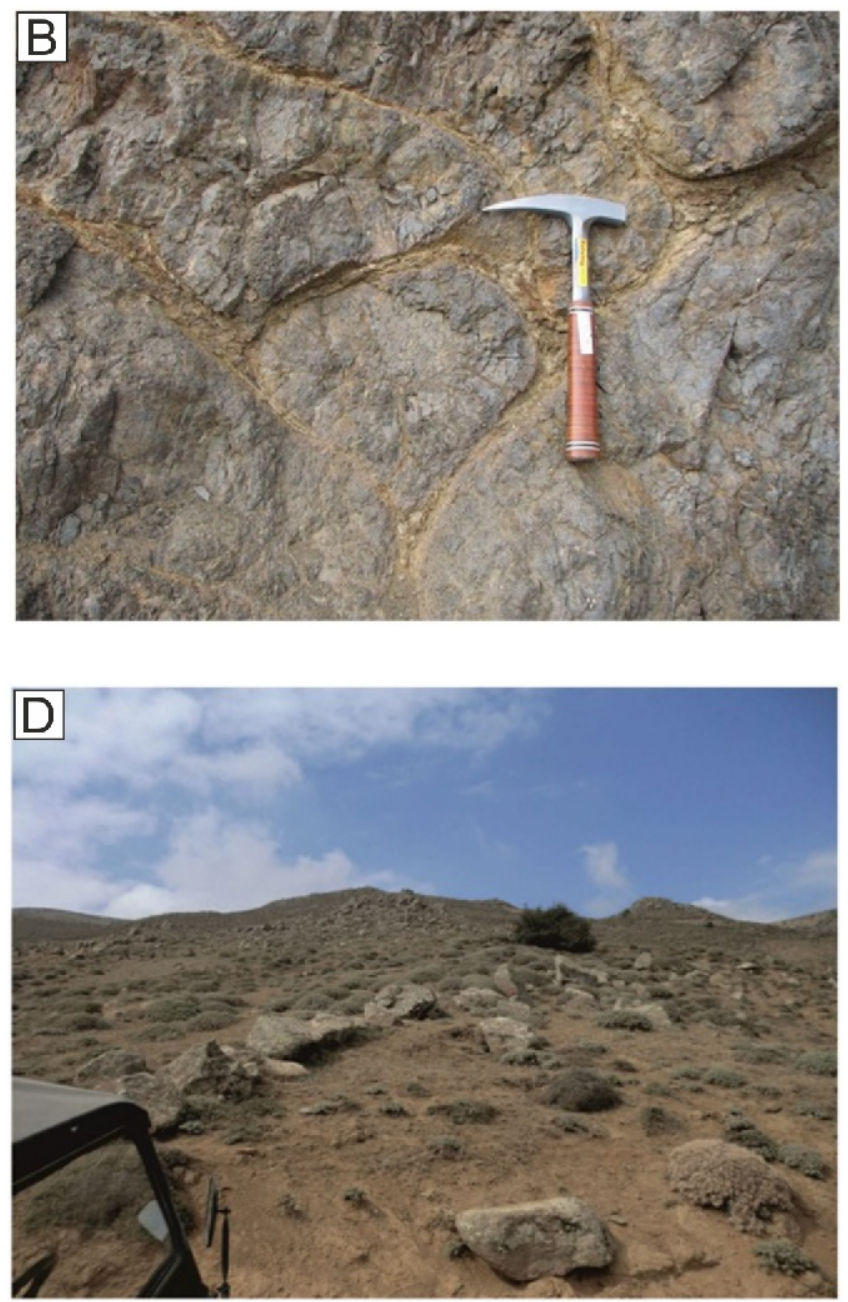

Fig. 3. Field photographs of major exposures of the Deylamn complex

A - alternating sheet lavas and the pelagic limestone beds; B - pillow structure in the basaltic pillow lava unit; C - small alkali rhyolite bodies; $\mathbf{D}$ - a gabbro body 
Representative major (wt.\%) and trace element (ppm) compositions of igneous rocks from the Deylaman igneous complex, northern Iran (samples specified with stars are from this study and others are from other studies Haghnazar and Malakotian, 2009; Haghnazar, 2012; Haghnazar et al., 2015, 2016)

\begin{tabular}{|c|c|c|c|c|c|c|c|c|c|c|c|c|c|c|c|c|c|c|}
\hline & \multicolumn{18}{|c|}{ Rock type } \\
\hline & \multicolumn{6}{|c|}{ Basalt (pillow lavas) } & \multicolumn{12}{|c|}{ Basalt (sheet lavas) } \\
\hline & \multicolumn{18}{|c|}{ Sample } \\
\hline & $\mathrm{SP} 1$ & SP19 & SP2 & $\mathrm{SP} 4$ & SP18 & SP16 & $\mathrm{N} 2^{*}$ & $\mathrm{~N}^{*}$ & $\mathrm{~N} 7^{*}$ & $\mathrm{N9*}$ & EB17 & EB18 & JB16 & JB13 & JB10 & JB3 & JB2 & JB27 \\
\hline $\mathrm{SiO}_{2}$ & 47.5 & 49.8 & 48.51 & 46.06 & 50.2 & 45.6 & 51.29 & 50.05 & 49.84 & 48.16 & 67 & 67.8 & 48.29 & 48.77 & 48.24 & 49.02 & 50.22 & 48.63 \\
\hline $\mathrm{TiO}_{2}$ & 2.48 & 1.93 & 2.43 & 2.7 & 1.97 & 2.09 & 1.05 & 1.06 & 1.09 & 1.16 & 0.44 & 0.42 & 1.10 & 0.97 & 0.94 & 1.17 & 0.95 & 1.19 \\
\hline $\mathrm{Al}_{2} \mathrm{O}_{3}$ & 14.28 & 13.37 & 14.98 & 13.6 & 13.07 & 14.15 & 15.37 & 15.23 & 14.98 & 14.55 & 15.1 & 14.5 & 15.42 & 17.69 & 15.87 & 16.11 & 14.16 & 15.05 \\
\hline $\mathrm{Fe}_{2} \mathrm{O}_{3}$ & 13.48 & 10.89 & 13.41 & 14.78 & 11.07 & 13.34 & 11.29 & 11.96 & 12.23 & 11.73 & 3.34 & 3.47 & 11.92 & 11.02 & 11.69 & 12.15 & 11.43 & 13.54 \\
\hline MnO & 0.7 & 0.21 & 0.2 & 0.24 & 0.18 & 0.27 & 0.21 & 0.18 & 0.2 & 0.27 & 0.03 & 0.06 & 0.13 & 0.11 & 0.12 & 0.13 & 0.12 & 0.15 \\
\hline $\mathrm{MgO}$ & 5.85 & 8.08 & 7.13 & 6.84 & 8.46 & 9.1 & 4.73 & 5.17 & 5.54 & 4.06 & 0.48 & 0.27 & 4.89 & 4.61 & 6.44 & 3.75 & 6.57 & 4.25 \\
\hline $\mathrm{CaO}$ & 8.24 & 7.53 & 8.25 & 9.05 & 7.66 & 9.67 & 7.65 & 7.54 & 6.55 & 10.48 & 2 & 2.86 & 8.91 & 9.37 & 9.51 & 8.56 & 9.67 & 8.72 \\
\hline $\mathrm{Na}_{2} \mathrm{O}$ & 2.12 & 1.67 & 2.41 & 1.94 & 1.58 & 1.55 & 1.84 & 1.76 & 2.26 & 1.5 & 5.18 & 4.24 & 2.25 & 2.35 & 2.64 & 2.33 & 2.10 & 2.84 \\
\hline $\mathrm{K}_{2} \mathrm{O}$ & 1.13 & 2.3 & 1.04 & 0.88 & 2.05 & 1.56 & 1.86 & 1.75 & 1.82 & 1.48 & 3.3 & 3.4 & 2.43 & 1.70 & 0.82 & 3.27 & 2.47 & 2.16 \\
\hline $\mathrm{P}_{2} \mathrm{O}_{5}$ & 0.34 & 0.33 & 0.415 & 0.42 & 0.32 & 0.28 & 0.36 & 0.39 & 0.39 & 0.38 & 0.11 & 0.16 & 0.35 & 0.24 & 0.22 & 0.36 & 0.21 & 0.28 \\
\hline L.O.I. & 3.64 & 3.8 & 1.06 & 3.32 & 3.24 & 2.26 & 3.86 & 4.41 & 4.63 & 5.69 & 2.28 & 1.34 & 3.34 & 2.68 & 3.05 & 2.58 & 1.77 & 2.87 \\
\hline Total & 99.76 & 99.91 & 99.83 & 99.83 & 99.8 & 99.87 & 99.51 & \begin{tabular}{|l}
99.49 \\
\end{tabular} & 99.52 & 99.45 & 99.26 & 98.52 & 99.03 & \begin{tabular}{|l}
99.51 \\
\end{tabular} & 99.54 & 99.43 & \begin{tabular}{|l|}
99.67 \\
\end{tabular} & 99.68 \\
\hline Cs & & & & & & & 10.2 & 11.4 & 12.7 & 12.6 & 2.2 & 1.5 & $<5$ & $<5$ & $<5$ & $<5$ & $<5$ & $<5$ \\
\hline $\mathbf{R b}$ & 5.3 & 26.4 & 33.3 & 1.2 & 47.3 & 13 & \begin{tabular}{|l|l|}
120.6 \\
\end{tabular} & 121.7 & 132.1 & 130.2 & 142 & 103 & 39.8 & 51.1 & 28.9 & 71.4 & 50.9 & 39.3 \\
\hline $\mathrm{Ba}$ & 164.1 & 306.3 & 206 & 185 & 331.2 & 218.4 & 263.1 & 237 & 293.9 & 216.9 & 1370 & 800 & \begin{tabular}{|l|}
357.1 \\
\end{tabular} & 391.7 & \begin{tabular}{|l|}
253.5 \\
\end{tabular} & 375.8 & 388.4 & 380.1 \\
\hline $\mathrm{Sr}$ & 433.2 & 311.9 & 359.3 & 343 & 321.3 & 442.5 & \begin{tabular}{|l|l|}
422.2 \\
\end{tabular} & 425.5 & 456.8 & 447.2 & 440 & 350 & \begin{tabular}{|l|l|}
600.2 \\
\end{tabular} & \begin{tabular}{|l|l|}
817.2 \\
\end{tabular} & \begin{tabular}{|l|l|}
715.9 \\
\end{tabular} & 687.9 & 639.5 & 612.1 \\
\hline $\mathrm{Pb}$ & $\begin{array}{l}16.7 \\
\end{array}$ & 14.1 & 19.4 & 8.1 & 15.9 & 15.7 & 11.5 & 8.8 & 10.4 & $<0.5$ & & & 8.4 & 8.1 & 7.8 & 8.2 & 7.8 & 8.6 \\
\hline Th & 5.2 & 8.8 & 5.5 & 4.1 & 5.8 & 4 & 7.6 & 7.4 & 9 & 7 & 14.1 & 14 & 2.20 & 3.90 & 2.90 & $<2$ & $<2$ & $<2$ \\
\hline$U$ & 8.9 & 6.1 & 7.8 & 6.3 & 6.8 & 7.2 & 4.6 & 5 & 5.1 & 5.1 & 2.32 & 3.17 & & & & & & \\
\hline $\mathrm{Zr}$ & 135.7 & 116.2 & 132.7 & 130.6 & 114.5 & 108.5 & 178.9 & 174 & 193.7 & 164 & 195 & 241 & \begin{tabular}{|l|}
140.9 \\
\end{tabular} & 153.6 & \begin{tabular}{|l|}
151.8 \\
\end{tabular} & 154.3 & 137.4 & 148.1 \\
\hline $\mathrm{Hf}$ & 5.6 & 3.1 & 2.5 & 6.1 & 3.6 & 2.3 & 3.5 & 3.7 & 4.4 & 3.6 & 5 & 6 & 7.2 & 4.6 & 4.4 & $<4$ & 11.3 & 5.8 \\
\hline $\mathrm{Ta}$ & 1.6 & 1.2 & 1.5 & 2.3 & 1.6 & 1.2 & 0.7 & 0.9 & 0.7 & 0.9 & 1.5 & 1.3 & $<5$ & $<5$ & $<5$ & $<5$ & $<5$ & $<5$ \\
\hline$Y$ & 23.7 & 29.8 & 23.1 & 21.6 & 28.7 & 25.8 & 22.4 & 22.2 & 25.6 & 22.3 & 26.7 & 18.1 & 31.0 & 29.5 & 24.7 & 34.3 & 30.2 & 30.3 \\
\hline $\mathrm{Nb}$ & 33.1 & 27.1 & 26.7 & 25.4 & 26.3 & 23.6 & 25.6 & 25.4 & 30.5 & 25.7 & 16 & 18 & 12.9 & 10.2 & 12.5 & 13.5 & 6.0 & 13.3 \\
\hline La & 18.9 & 17.1 & 16 & 15.7 & 17.3 & 11.6 & 15.6 & 14.7 & 17.3 & 14.8 & 63 & 48.7 & & & & & & \\
\hline $\mathrm{Ce}$ & 28 & 23.9 & 27.1 & 20.8 & 22.9 & 31.2 & 42.6 & 43.3 & 52.1 & 42.3 & 91.8 & 84.2 & 40.2 & 37.5 & 31.9 & 35.9 & 39.3 & 35.0 \\
\hline $\mathrm{Pr}$ & 6.7 & 6.2 & 6.5 & 6.8 & 6.1 & 6.2 & 5.8 & 5 & 5.8 & 5 & 10.1 & 9.33 & & & & & & \\
\hline $\mathrm{Nd}$ & 84.1 & 34.8 & 74.1 & 72 & 36 & 34.8 & 38.4 & 37 & 42.7 & 36.4 & 34.2 & 31.1 & 37.0 & 7.3 & 15.0 & 31.2 & 18.2 & 38.9 \\
\hline $\mathrm{Sm}$ & 5.4 & 4.7 & 4.6 & 4.2 & 4.9 & 4.4 & 3.7 & 4.2 & 4.7 & 4.2 & 6.1 & 5 & & & & & & \\
\hline $\mathrm{Eu}$ & 1.1 & 0.7 & 1.2 & 0.9 & 0.8 & 0.9 & 1.5 & 1.6 & 1.8 & 1.5 & 1.55 & 1.15 & $<3$ & $<3$ & $<3$ & $<3$ & $<3$ & $<3$ \\
\hline Gd & 5.6 & 4.6 & 5.3 & 5.1 & 4.5 & 4.8 & 4.4 & 4.3 & 5.2 & 4.3 & 5.19 & 3.86 & & & & & & \\
\hline Tb & 3.7 & 1.3 & 3.3 & 3.4 & 1.4 & 1.5 & 1.8 & 1.8 & 2.3 & 1.9 & 0.81 & 0.56 & 1.1 & 1.1 & 1.1 & 1.1 & 1.1 & 1.2 \\
\hline Dy & 7.1 & 2.6 & 3 & 3.5 & 3 & 3.4 & 4 & 3.6 & 4 & 3.9 & 4.67 & 3.23 & & & & & & \\
\hline Ho & 0.5 & 0.5 & 0.5 & 0.5 & 0.5 & 0.5 & 0.6 & 0.6 & 0.6 & 0.6 & 0.94 & 0.66 & & & & & & \\
\hline $\mathrm{Er}$ & 4.1 & 5.3 & 5 & 4 & 3.7 & 5.3 & 1.3 & 0.5 & 1.1 & $<0.5$ & 2.67 & 1.81 & & & & & & \\
\hline $\mathrm{Tm}$ & 0.2 & 0.3 & 0.3 & 0.3 & 0.2 & 0.2 & 0.4 & 0.3 & 0.4 & 0.3 & 0.39 & 0.28 & & & & & & \\
\hline $\mathrm{Yb}$ & 2.6 & 2.4 & 2.2 & 2.2 & 2.5 & 2.6 & 2.6 & 2.6 & 2.9 & 2.6 & 2.6 & 1.8 & & & & & & \\
\hline Lu & 0.4 & 0.3 & 0.4 & 0.3 & 0.3 & 0.4 & 0.8 & 1 & 1 & 0.7 & 0.34 & 0.22 & & & & & & \\
\hline Sc & 21.2 & 23.3 & 18.2 & 19.5 & 24.9 & 30.1 & 31.5 & 33.8 & 38.4 & 32.4 & & & 27.5 & 26.5 & 29.3 & 27.6 & 31.2 & 26.6 \\
\hline $\mathrm{Cr}$ & 123.7 & 99.9 & 126.2 & 139.4 & 81.5 & 55.8 & 77 & \begin{tabular}{|l|}
72.5 \\
\end{tabular} & 85.8 & 64.2 & & & 28.8 & $15 / 7$ & 41.5 & $<10$ & \begin{tabular}{|l|}
141.0 \\
\end{tabular} & 19.5 \\
\hline $\mathrm{Ni}$ & 112.6 & 130.5 & 56.9 & 254.4 & 55.2 & 239.9 & 16.1 & 16.7 & 17.8 & 14.9 & 11 & 19 & $<5$ & $<5$ & $<5$ & $<5$ & 35.5 & $<5$ \\
\hline Co & 61.9 & 39.6 & 48.3 & 34.7 & 39.9 & 36.7 & 34.8 & 31.5 & 39.1 & 32.9 & 6.4 & 4.8 & 37.7 & 35.0 & 38.0 & 38.9 & 38.0 & 43.9 \\
\hline $\mathbf{v}$ & 200 & 178.8 & 171.5 & 170.6 & 187.5 & 227.7 & 233 & 236.3 & 279.5 & 232.3 & 42 & 29 & \begin{tabular}{|l|}
259.2 \\
\end{tabular} & 221.9 & \begin{tabular}{|l|}
203.2 \\
\end{tabular} & 242.1 & 245.0 & 268.2 \\
\hline $\mathrm{Ga}$ & & & & & & & 18 & \begin{tabular}{|l|}
16.2 \\
\end{tabular} & 19 & 14 & 17 & 17 & 19.9 & 21.9 & 20.8 & 21.1 & 20.7 & 19.9 \\
\hline$Z n$ & 107.4 & 73.6 & 90.5 & 89.8 & 73.1 & 76.7 & 78.7 & 74 & 85.9 & 75.4 & & & 86.1 & 97.9 & 80.0 & 99.1 & 97.9 & 114.8 \\
\hline $\mathrm{Cu}$ & 65.5 & 60.5 & 52.7 & 53.3 & 63.1 & 86.3 & 51.3 & 49.6 & 60.3 & 47.8 & 18 & 11 & \begin{tabular}{|l|}
123.4 \\
\end{tabular} & 112.5 & 47.6 & $134.8^{*}$ & $141.1^{*}$ & $148.7^{*}$ \\
\hline $\mathrm{Be}$ & & & & & & & 1.6 & 1.3 & 1.6 & 1.3 & & & & & & & & \\
\hline
\end{tabular}


Tab. 1 cont.

\begin{tabular}{|c|c|c|c|c|c|c|c|c|c|c|c|c|c|c|c|c|}
\hline & \multicolumn{16}{|c|}{ Rock type } \\
\hline & \multicolumn{4}{|c|}{ Rhyolite } & \multicolumn{4}{|c|}{ Trachyte } & \multicolumn{8}{|c|}{ Intrusions } \\
\hline & \multicolumn{16}{|c|}{ Sample } \\
\hline & $N 6 A^{*}$ & $\mathrm{~N} 6 \mathrm{C}^{*}$ & EB24 & EB29 & $\mathrm{N} 12^{*}$ & N15* & EB20 & EB31 & N6B* & $\mathrm{N} 13^{*}$ & J-G5 & J-G11 & J-G8 & J-D30 & $\mathrm{j}-\mathrm{d} 30 \mathrm{a}$ & J-G21a \\
\hline $\mathrm{SiO}_{2}$ & 72.05 & 67.03 & 73.7 & 69.5 & 61.98 & 73.01 & 59.8 & 63.5 & 69.08 & 47.1 & 49.03 & 45.13 & 45.78 & 49.73 & 50.97 & 47.59 \\
\hline $\mathrm{TiO}_{2}$ & 0.47 & 0.9 & 0.26 & 0.36 & 0.53 & 0.35 & 0.22 & 0.67 & 0.74 & 3.16 & 0.94 & 1.31 & 1.15 & 1.007 & 0.925 & 1.254 \\
\hline $\mathrm{Al}_{2} \mathrm{O}_{3}$ & 13.29 & 15.4 & 11.9 & 13.9 & 16.53 & 12.86 & 17.6 & 16.8 & 14.07 & 16.13 & 14.78 & 17.55 & 16.5 & 15.76 & 18.29 & 12.7 \\
\hline $\mathrm{Fe}_{2} \mathrm{O}_{3}$ & 3.04 & 3.89 & 3.89 & 3.61 & 7.11 & 3.2 & 5.8 & 3.04 & 3.96 & 13.8 & 12.15 & 12.57 & 11.17 & 12.73 & 9.31 & 12.25 \\
\hline $\mathrm{MnO}$ & 0.11 & 0.18 & 0.06 & 0.04 & 0.17 & 0.13 & 0.17 & 0.14 & 0.17 & 0.18 & 0.12 & 0.22 & 0.17 & 0.175 & 0.124 & 0.222 \\
\hline $\mathrm{MgO}$ & 0.47 & 0.45 & 0.03 & 0.26 & 0.56 & 0.23 & 0.2 & 0.11 & 0.75 & 2.77 & 5.08 & 8.55 & 8.23 & 5.02 & 4.76 & 10.81 \\
\hline $\mathrm{CaO}$ & 1.31 & 1.46 & 0.09 & 0.34 & 1.06 & 0.92 & 1.5 & 0.69 & 2.2 & 7.07 & 13.42 & 10.91 & 13.59 & 11.4 & 10.33 & 12.45 \\
\hline $\mathrm{Na}_{2} \mathrm{O}$ & 3.51 & 2.96 & 4.67 & 4.26 & 3.7 & 3.76 & 5.61 & 8.47 & 3.29 & 3.25 & 1.78 & 1.45 & 1.11 & 1.74 & 2.41 & 1.24 \\
\hline $\mathrm{K}_{2} \mathrm{O}$ & 3.68 & 6.26 & 3.3 & 4.9 & 4.92 & 4.01 & 6.6 & 4 & 4.1 & 1.65 & 0.95 & 0.63 & 1.07 & 0.81 & 1.12 & 0.78 \\
\hline $\mathrm{P}_{2} \mathrm{O}_{5}$ & 0.17 & 0.42 & 0.02 & 0.07 & 0.2 & 0.1 & 0.11 & 0.16 & 0.34 & 0.97 & 0.23 & 0.13 & 0.11 & 0.133 & 0.221 & 0.068 \\
\hline L.O.I. & 1 & 0.55 & 0.94 & 1.1 & 2.73 & 0.94 & 1.43 & 0.45 & 0.7 & 3.2 & 1.24 & 1.02 & 1.14 & 1.26 & 1.2 & 0.59 \\
\hline Total & 99.1 & 99.49 & 98.86 & 98.34 & 99.48 & 99.51 & 99.04 & 98.03 & 99.41 & 99.28 & 99.72 & 99.47 & 100.02 & 99.77 & 99.66 & 99.95 \\
\hline Cs & 3.9 & 3.5 & 0.7 & 1.1 & 8.2 & 1.9 & 1.6 & 0.6 & 3.6 & 11.4 & & & & & & \\
\hline $\mathbf{R b}$ & 98.4 & 89 & 150 & 133 & 108.3 & 54.3 & 69.1 & 165 & 110.6 & 276 & 27 & 14 & 19 & 29 & 22 & 19 \\
\hline $\mathrm{Ba}$ & 482.3 & 777.9 & 80 & 250 & 715.2 & 425.8 & 430 & 540 & 953.3 & 494.1 & 179 & 51 & 81 & 88 & 282 & 249 \\
\hline $\mathrm{Sr}$ & 363.6 & 331.9 & 90 & 150 & 387 & 198.6 & 110 & 240 & 400 & 1006.1 & 460 & 574 & 555 & 522 & 634 & 444 \\
\hline $\mathrm{Pb}$ & 3.3 & 3.1 & & & 8.7 & 6.7 & & & 2.6 & 2.9 & 13 & 14 & 16 & 4 & 6 & 16 \\
\hline Th & 5 & 6.1 & 20 & 18.9 & 8.1 & 6.1 & 14.1 & 11.6 & 4.5 & 11.9 & 3 & 4 & 2 & 5 & 2 & 2 \\
\hline U & 1.5 & 2 & 2.69 & 4.44 & 3.4 & 0.9 & 4.61 & 2.51 & 1.4 & 5.1 & 1 & 3 & 1 & 1 & 1 & 1 \\
\hline $\mathrm{Zr}$ & 258.6 & 892.3 & 505 & 581 & 1069. & 1034.3 & 1000 & 427 & 226.9 & 428.9 & 89 & 83 & 80 & 80 & 97 & 74 \\
\hline $\mathrm{Hf}$ & 1.2 & 2 & 11 & 15 & 2.6 & 1.7 & 20 & 10 & 1.7 & 6 & & & & & & \\
\hline Ta & $<0.1$ & 0.2 & 7.7 & 7 & 0.5 & $<0.1$ & 7.3 & 4.2 & 0.1 & 0.9 & & & & & & \\
\hline$Y$ & 42.3 & 42.7 & 52.9 & 44.6 & 38.2 & 51 & 33.8 & 28.2 & 39.5 & 34.7 & 12 & 12 & 15 & 15 & 16 & 14 \\
\hline $\mathrm{Nb}$ & 18 & 35.1 & 118 & 108 & 17.8 & 16.7 & 112 & 71 & 27.3 & 110.7 & 14 & 10 & 13 & 11 & 13 & 12 \\
\hline La & 72.1 & 63.9 & 43.6 & 75 & 88.6 & 77 & 98.4 & 67 & 65 & 36.7 & & & & & & \\
\hline $\mathrm{Ce}$ & 137.6 & 132.9 & 172 & 145 & 165 & 157 & 167 & 114 & 129.1 & 96.2 & & & & & & \\
\hline $\mathrm{Pr}$ & 9.8 & 7.8 & 7.89 & 16.7 & 10.8 & 9.5 & 16.9 & 13.6 & 8.3 & 15.9 & & & & & & \\
\hline $\mathrm{Nd}$ & 45.6 & 64.8 & 25 & 59.6 & 46.2 & $\begin{array}{l}50.8 \\
\end{array}$ & 51.2 & 49.4 & 55 & 129 & & & & & & \\
\hline $\mathrm{Sm}$ & 7.7 & 7.4 & 4.8 & 11.4 & 6.1 & 8.8 & 7.7 & 9 & 8.1 & 7.7 & & & & & & \\
\hline $\mathrm{Eu}$ & 1.6 & 2.6 & 0.42 & 1.3 & 1.6 & 1.2 & 1.44 & 2.52 & 2.7 & 3.4 & & & & & & \\
\hline Gd & 3.6 & 4.2 & 5.26 & 0.39 & 4.1 & 4.4 & 5.55 & 7.08 & 3.9 & 7.3 & & & & & & \\
\hline $\mathrm{Tb}$ & 1.1 & 2.3 & 1.25 & 1.56 & 1.2 & 1.1 & 0.95 & 1.11 & 1.6 & 8.1 & & & & & & \\
\hline Dy & 6.7 & 7.6 & 9.2 & 8.69 & 5.3 & 8.3 & 5.88 & 6.22 & 6.4 & 5.7 & & & & & & \\
\hline Ho & 1.2 & 1.2 & 2 & 1.7 & 1.3 & 1.5 & 1.24 & 1.16 & 1.3 & 1.5 & & & & & & \\
\hline $\mathrm{Er}$ & 1.5 & 1.6 & 5.68 & 4.76 & 1.4 & 2 & 3.66 & 2.95 & 1.2 & 1.4 & & & & & & \\
\hline $\mathrm{Tm}$ & 0.1 & 0.2 & 0.89 & 0.71 & 0.2 & 0.1 & 0.58 & 0.44 & 0.1 & 0.4 & & & & & & \\
\hline $\mathrm{Yb}$ & 2.9 & 3.1 & 5.6 & 4.4 & 3.2 & 3.5 & 4 & 2.8 & 2.3 & 3.1 & & & & & & \\
\hline Lu & 0.4 & 0.5 & 0.73 & 0.58 & 0.7 & 0.4 & 0.59 & 0.35 & 0.4 & 1.1 & & & & & & \\
\hline $\mathrm{Sc}$ & 5.4 & 11.2 & & & 3.7 & 4.4 & & & 9.2 & 24.3 & & & & & & \\
\hline $\mathrm{Cr}$ & 38.2 & 48.7 & & & 36.3 & 46.8 & & & 45.7 & 93.2 & 66 & 59 & 54 & 62 & 47 & 115 \\
\hline $\mathrm{Ni}$ & 21.8 & 7 & 10 & 33 & 2 & 3.9 & 7 & 17 & 8 & 29.7 & 54 & 71 & 59 & 34 & 19 & 45 \\
\hline Co & 5.9 & $<0.5$ & 0.7 & 1.6 & 0.6 & $<0.5$ & 1.2 & 4.1 & $<0.5$ & 40.1 & 78 & 95 & 65 & 45 & 32 & 53 \\
\hline $\mathbf{v}$ & 13.2 & 27.3 & 5 & 6 & 19.3 & 7.4 & 5 & 21 & 14.2 & 293.6 & 354 & 383 & 377 & 377 & 354 & 258 \\
\hline Ga & 21.9 & 22.2 & 26 & 27 & 22.7 & 23.4 & 29 & 26 & 24.1 & 25 & & & & & & \\
\hline $\mathrm{Zn}$ & 92.7 & $\begin{array}{l}138.6 \\
\end{array}$ & & & 106.6 & 145 & & & 99.3 & 126.2 & 99 & 121 & 130 & 66 & 56 & 67 \\
\hline $\mathrm{Cu}$ & 3.5 & 3.1 & 8 & 9 & 5.3 & 3.7 & 12 & 35 & 1 & 63.5 & 170 & 190 & 220 & 153 & 149 & 41 \\
\hline $\mathrm{Be}$ & 6.2 & 4.5 & & & 5 & 5.6 & & & 4.4 & 1.8 & & & & & & \\
\hline
\end{tabular}



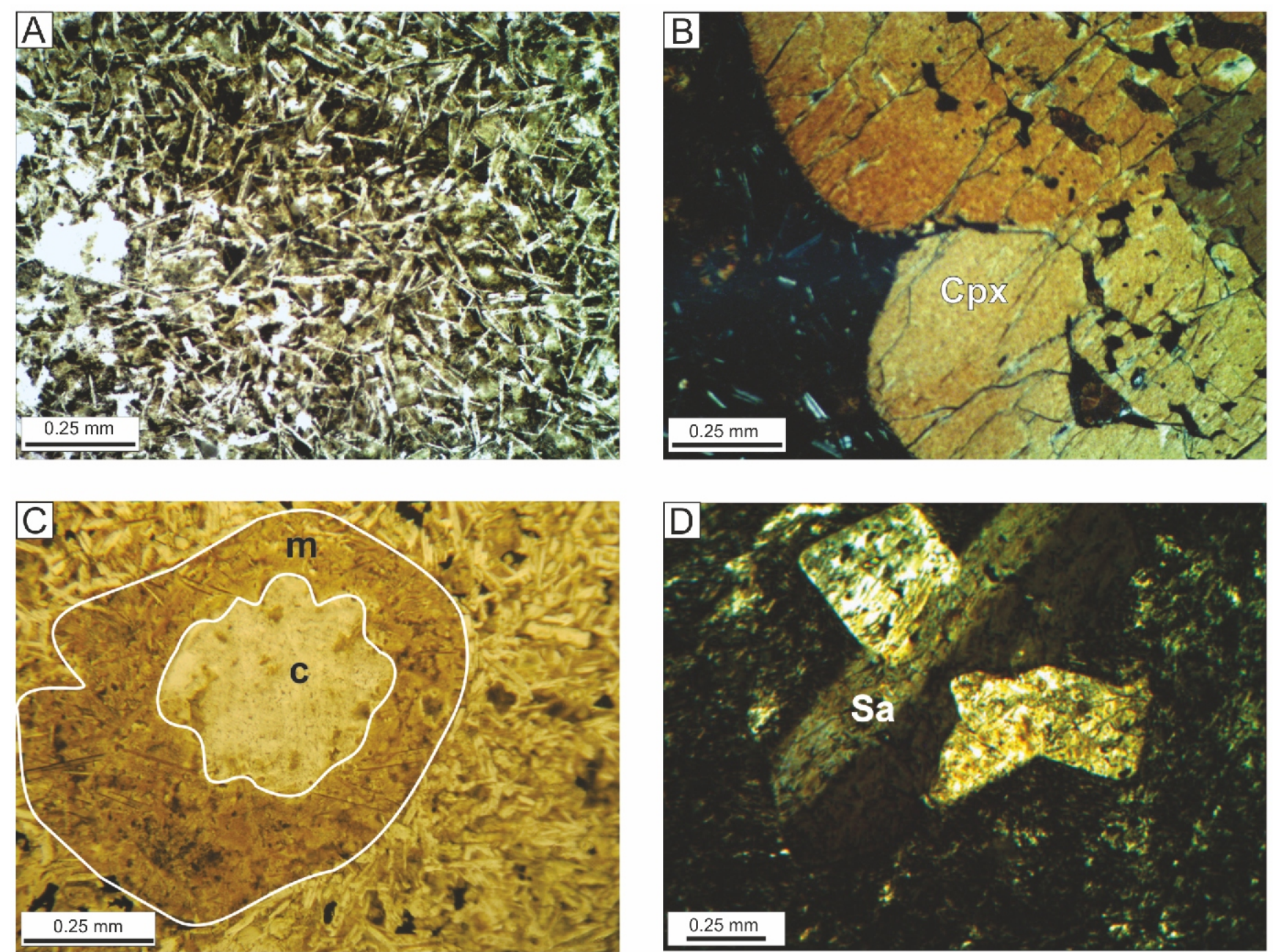

Fig. 4. Microphotographs of A - intersertal to hyalo-aphanitic texture of the pillow lavas (XPL); B - corroded cumulophyric phenocrysts of clinopyroxene (Cpx) in the sheet lavas (XPL); $C$ - segregation vesicles in the basaltic sheet lavas in which the vesicles were lined by a darker colored melt $(\mathrm{m})$ than the groundmass and then filled by secondary calcite (c) (PPL); $D$ - the sanidine phenocrysts (Sa) with a synneusis junction in the alkali trachyte (XPL)

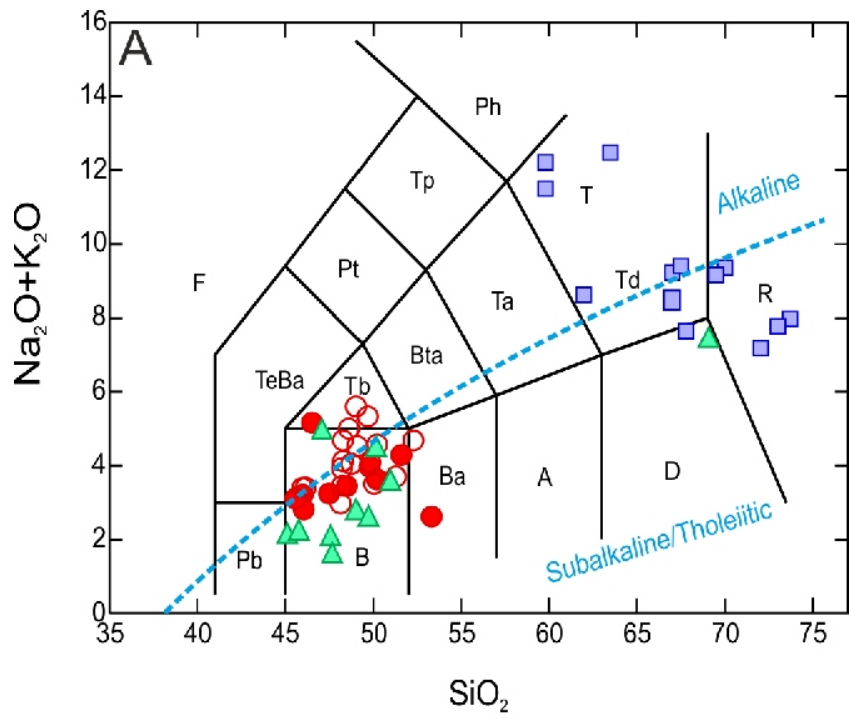

o pillow lavas

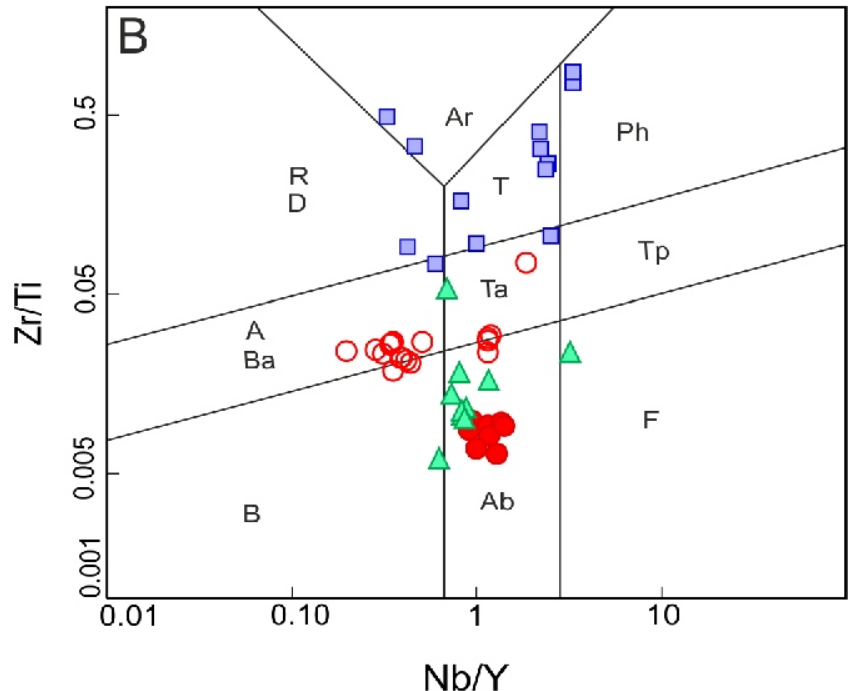

$\triangle$ intrusions

$\square$ felsic lavas

Fig. 5. Chemical compositions of rock samples from the Deylamn district in (A) total alkali-silica diagram (Le Maitre et al., 2002) and (B) Zr/Ti vs. Nb/Y diagram of Winchester and Floyd (1977) modified by Pearce (1996)

A - andesite; Ab - alkali basalt; Ar - alkali rhyolite; B - basalt; Ba - basaltic andesite; Bta - basaltic trachyandesite; $\mathrm{D}$ - dacite;

$\mathrm{F}$ - foidite; $\mathrm{Pb}$ - picrobasalt; $\mathrm{Ph}$ - phonolite; $\mathrm{Pt}$ - phonotephrite; $\mathrm{R}$ - rhyolite; $\mathrm{T}$ - trachyte; $\mathrm{Ta}$ - trachyandesite; $\mathrm{Tb}$ - trachybasalt;

$\mathrm{Td}$ - trachydacite; Te/Ba - tephrite/basanite; Tp - tephriphonolite 
The Harker (1909) variation diagrams of many major and trace elements (e..g, $\mathrm{Al}_{2} \mathrm{O}_{3}, \mathrm{Fe}_{2} \mathrm{O}_{3}{ }^{\mathrm{t}}, \mathrm{CaO}, \mathrm{Na}_{2} \mathrm{O}, \mathrm{Ce}, \mathrm{Cr}, \mathrm{Rb}$; Fig. 6) show two prominent characteristics: (1) a clear bimodal distribution of mafic and felsic samples and or the relative rarity of intermediate ones $\left(\mathrm{SiO}_{2}=53.32-59.8 \mathrm{wt} . \%\right)$; (2) evolutionary trends in the plots; for example, ascending trends of $\mathrm{Na}_{2} \mathrm{O}, \mathrm{Ce}$, $\mathrm{Y}, \mathrm{Rb}$; descending trends of $\mathrm{Fe}_{2} \mathrm{O}_{3}, \mathrm{CaO}, \mathrm{Cr}$; and also an ascending and then descending trend of $\mathrm{Al}_{2} \mathrm{O}_{3}$ (Fig. 6). Thus, it may be proposed that such trends were formed by some magmatic evolutionary processes such as fractional crystallization.

All the samples in the REE spider diagrams normalized to chondrites (Boynton, 1984) show a negative slope from LREEs to HREEs (Fig. 7A). However, the basaltic rocks (both pillow lavas and sheet lavas) have a more flat pattern $[(\mathrm{La} / \mathrm{Yb}) \mathrm{n}$ $=3.93-4.16]$ relative to the felsic rocks $[(\mathrm{La} / \mathrm{Yb}) \mathrm{n}=10.92]$. The $(\mathrm{La} / \mathrm{Yb}) \mathrm{n}$ ratio for EMORBs and OIBs is 1.91 and 12.92 respectively (data from Sun and McDonough, 1989). Although there are some anomalies due to the effects of the subduction processes (e.g., positive anomalies for $\mathrm{K}$ and $\mathrm{Th}$ and negative ones for $\mathrm{Ti}, \mathrm{Nb}$ and $\mathrm{Ta}$ ), there is seen a clear similarity between the patterns of the basaltic samples and OIBs as well as a distinctive bulge in LILEs (Sr-Ba) in the multi-element spider diagrams (Fig. 7B).

\section{TECTONIC SETTING}

The samples show (Fig. 8A-C) variable situations between within-plate and the volcanic arc settings. Moreover, the samples in the $\mathrm{Nb}(\mathrm{n}) / \mathrm{Th}(\mathrm{n})$ diagram of Saccani (2015; Fig. 8D) show two important characteristics: (1) their position in a rift setting that changes into a convergent margin setting; (2) there are two distinct trends in the samples: supra-subduction zone enrichment (SSZ-E) and plume type or ocean island type enrichment (OIB-CE).

\section{DISCUSSION}

Although most of the Cretaceous volcanic events in Iran mainly occurred in the Mesozoic magmatic arc of the SSMA, many authors (e.g., Verdel et al., 2011) propose an episode of magmatic quiescence in Cretaceous before the Eocene volcanic flare-up. The Cretaceous magmatic activities in the Alborz zone are remarkable (e.g., Doroozi et al., 2016) as the following two cases: (1) as basaltic lavas underlain by limestones of the lower Cretaceous Tizkuh Formation (Annells et al., 1975; Taki, 2017; Jafari Sough et al., 2018), or interlayered with limestones of the Cretaceous Chalus Formation (Yadollah-Pour et al., 2019); and (2) the 98 Ma Nusha pluton in the central Alborz area (Lam, 2002). Moreover, as shown in Figure 1, all the Cretaceous igneous outcrops are seen in the vicinity of the Eocene volcanics.

The Cretaceous magmatism in the Alborz zone is envisaged as the product of a tensional/back-arc basin by many researchers (e.g., Alavi, 1996; Allen et al., 2006) or as parts of an ophiolitic sequence that formed in slow-spreading mid-oceanic ridges (Salavati et al., 2013). On the other hand, a within-plate continental rift has been postulated by Haghnazar and Malakotian (2009), Haghnazar (2012) and Haghnazar et al. (2015) for the same volcanic succession.

The Deylaman igneous Complex (DIC), located in the northern Alborz zone and behind the Eocene volcanics of the Alborz zone (Fig. 1A,B), is one of the few Cretaceous volcanic outcrops in the area. Therefore, consideration of the tectonomagmatic and petrological characteristics of the DIC is important in determining whether the events of the Cretaceous magmatism are associated with the large-scale Eocene events in the Alborz zone.

\section{MAGMA GENESIS}

As noted above, the DIC shows a compositional bimodality (Figs. 5 and 6). Moreover, the samples seem to have an affinity to OIBs and they may be proposed as the partial melts of deep sources. The main evidence for this suggestion is as follows: the negative slope of LREEs/HREEs ratio especially in the felsic samples (Fig. 7A); the relative similarity between the patterns of the basaltic samples and those of OIBs; the distinctive bulge in LILEs ( $\mathrm{Sr}-\mathrm{Ba}$ ) in the multi-element spider diagrams (Fig. 7B); and especially the occurrence of mafic samples between EMORBs and OIBs (Fig. 7A). Thus, it may be deduced that the mafic samples were likely the partial melts of EMORB sources. According to Winter (2014), EMORBs and OIBs are distinct from NMORBs and appear to originate in an enriched mantle reservoir. The same result is obtained in the multi-element spider diagrams (Fig. 7B); i.e., the close similarity between the patterns of the basaltic samples and OIBs. On the other hand, Winter (2014) suggests that the distinctive bulge in LILEs (Sr-Ba) is a typical characteristic of melts generated from relatively undepleted mantle in intraplate settings (e.g., Winter, 2014). Thus, they may be proposed as partial melts from deep undepleted mantle sources in a within-plate regime (Figs. 7 and 8). Also, Xia et al. (2012) indicate that the ratio of $\mathrm{Nb} / \mathrm{La}$ in island arc basalts (IABs) is $<0.8$. While this ratio for the felsic rocks is $\sim 0.33$ in average, the mafic rocks show a content of 1.97 in average. Thus, it may be concluded that subduction processes did not likely have a dominant role in the petrogenesis of the mafic rocks.

Furthermore, the plotted samples in the $\mathrm{Sr} / \mathrm{Th}$ vs. Th diagram (Fig. 9) show a meaningful trend in which the mafic samples coincide with fluid enrichment that emanated from fractional crystallization. According to Zou et al. (2008), Ba and Sr are highly mobile in fluids, whereas Th is much less mobile. Thus fluid-induced melting would produce lavas with high $\mathrm{Ba} / \mathrm{Th}$ and $\mathrm{Sr} / \mathrm{Th}$ ratios. However, Th, as a crustal component, shows more enrichment in the felsic lavas.

On the other hand, the occurrence of the mafic samples on an evolving trend from an OIB source (for pillow lavas) to an EMORB source (for sheet lavas) in Figure $10 \mathrm{~A}$ provides a deep source for pillow lavas compared to a shallower source for the sheet lavas. Also, the pillow lavas show La/Yb $(=4.5-7.3)$ and $\mathrm{Zr} / \mathrm{Nb}(=4-5)$ ratios similar to those of OITs (oceanic island tholeiites; La/Yb $\sim 4)$ and OIBs $(\mathrm{Zr} / \mathrm{Nb}>10)$, respectively. The values for the sheet lavas have different ranges $(\mathrm{La} / \mathrm{Yb}=6-27$; $\mathrm{Zr} / \mathrm{Nb}=7.23$ ) implying evolutionary magmatic processes.

The plotted samples on the $\mathrm{Rb}$ vs. $\mathrm{Rb} / \mathrm{Yb}$ modeling diagram of Özdemir et al. (2006; Fig. 10B) show a good linear trend that originated from a proposed spinel Iherzolite source. Thus, it may be inferred that the parental magma originated from relatively deep sources (probably spinel Iherzolite) and then was evolved by AFC (assimilation-fractional crystallization) processes.

\section{VOLCANO-SEDIMENTARY ENVIRONMENT}

The Cretaceous lithological succession (Fig. 2) in the DIC consists of major geological units of basaltic pillow and sheet 

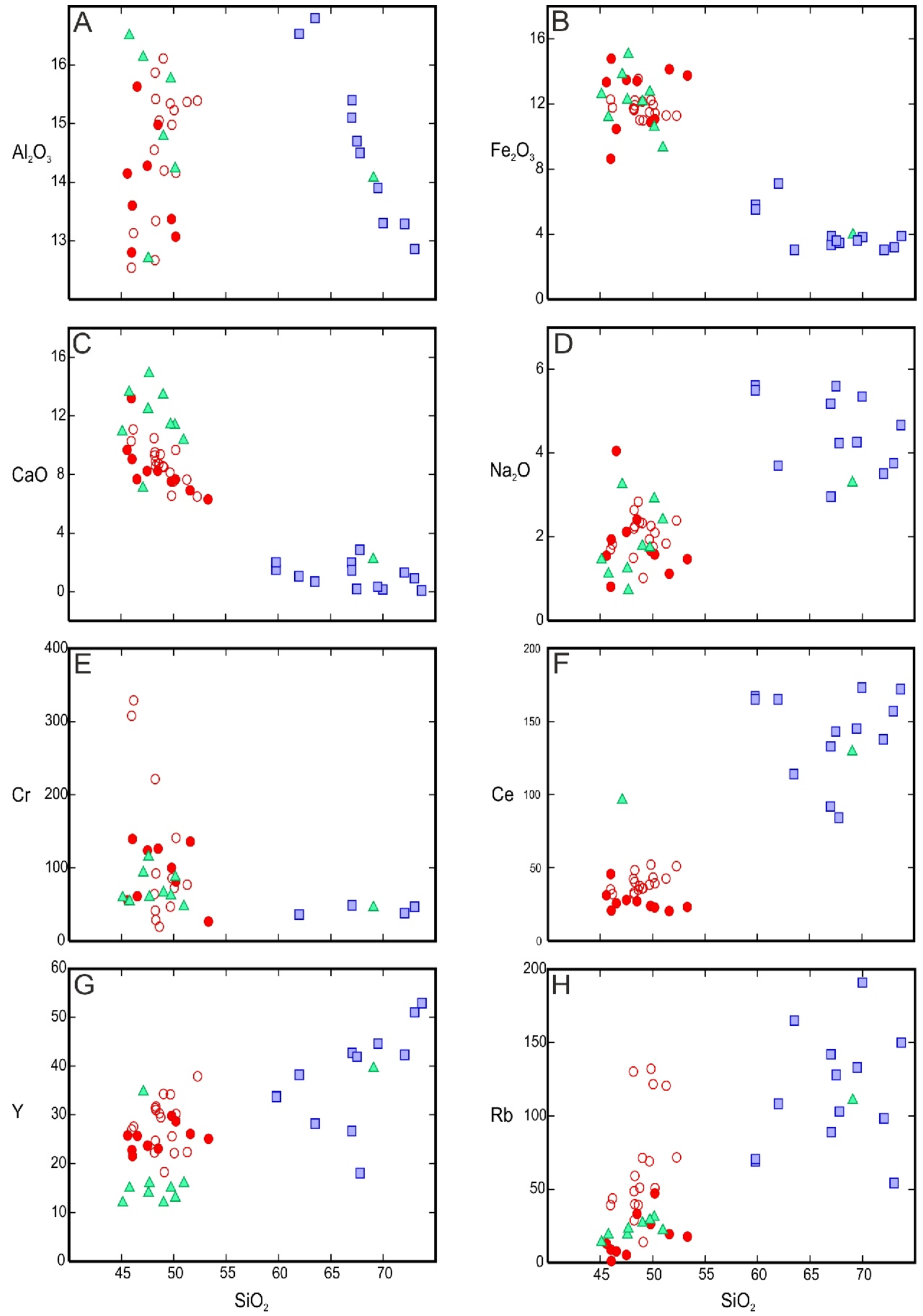

Fig. 6. Harker (1909) diagrams (wt.\% for oxides and ppm for elements) showing data for rock samples from the study area Note the bimodal distribution and the possible trends of the samples; symbols are the same as in Figure 5 

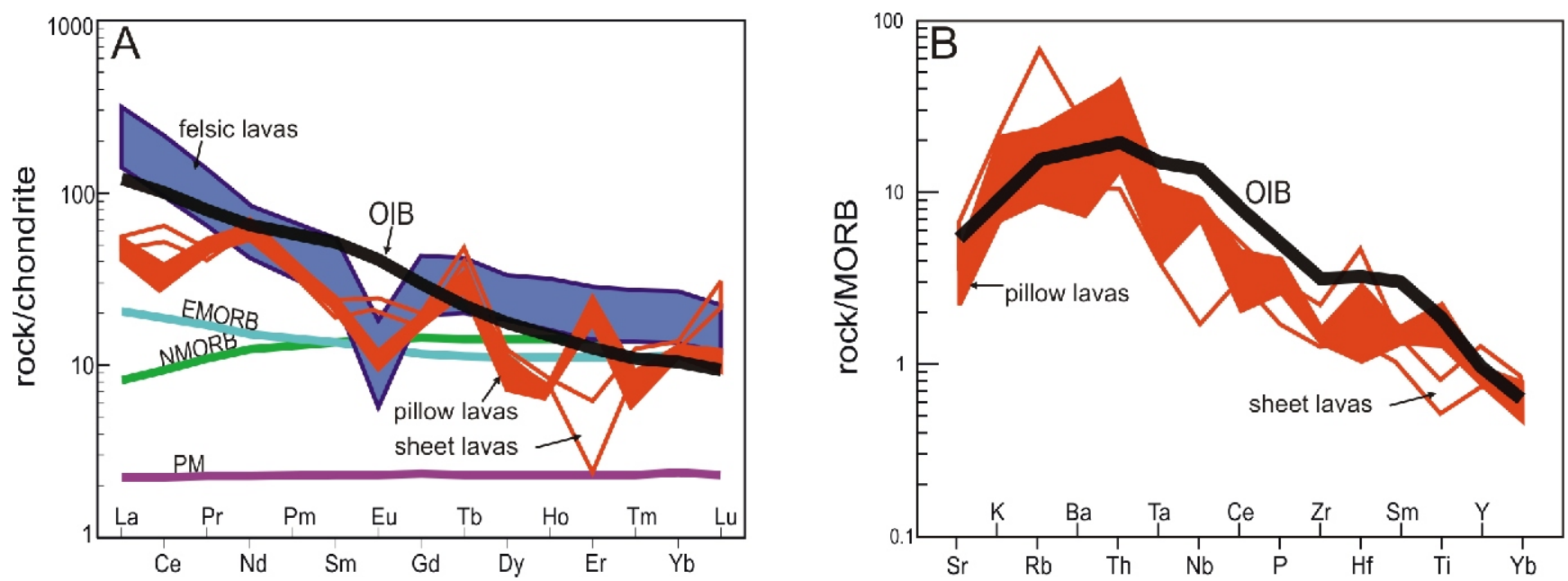

Fig. 7. Spider diagrams of the samples from the study area (A) REE pattern normalized to chondrite (Boynton, 1984), data of EMORB, NMORB, PM and OIB from Sun and McDonough (1989) and (B) multi-element pattern normalized to MORB (Pearce, 1983), OIB line from Winter (2014)

EMORB - enriched mid-ocean ridge basalts; NMORB - normal mid-ocean ridge basalts; OIB - ocean island basalts; PM - primitive mantle
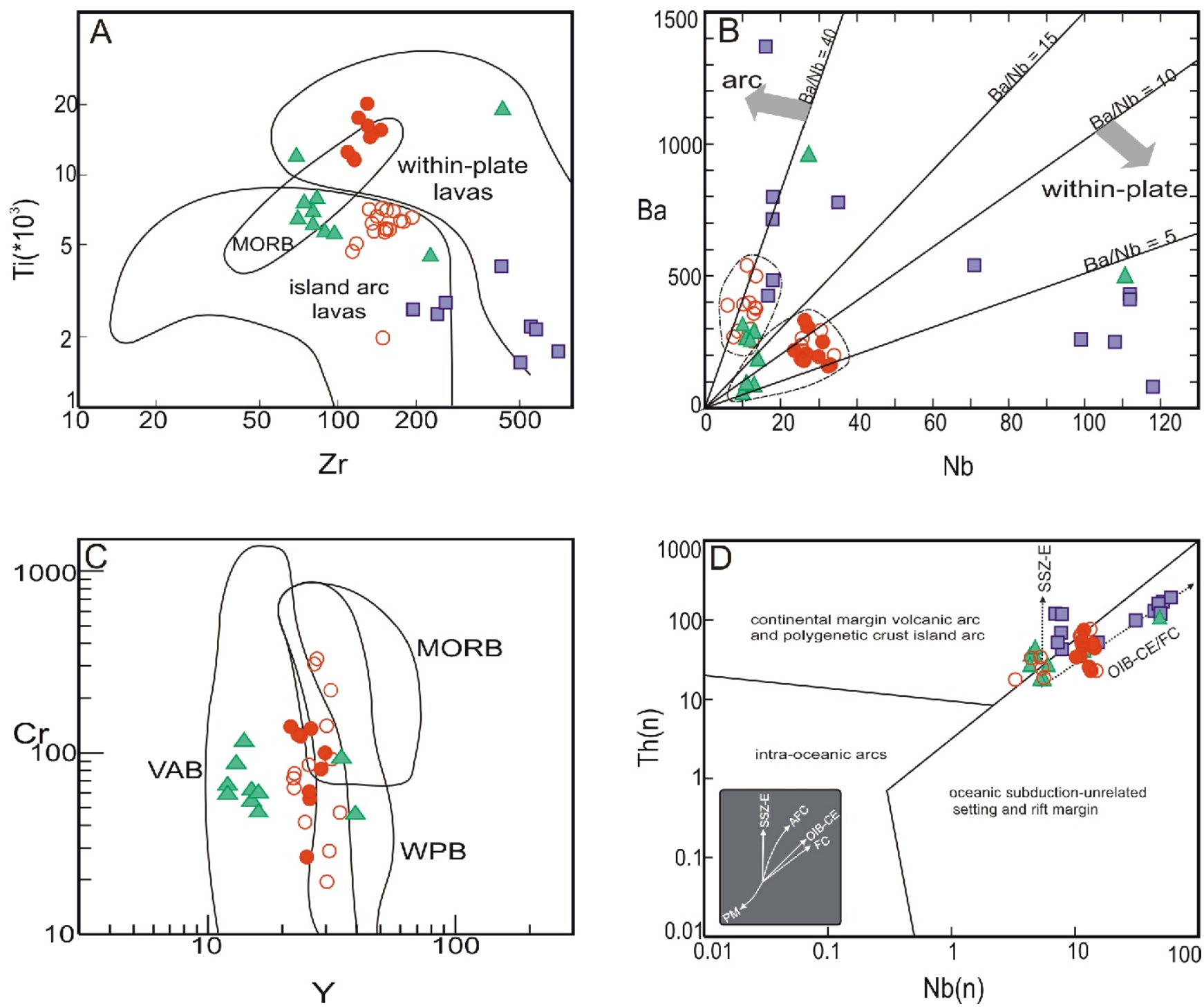

Fig. 8. Tectonomagmatic discrimination diagrams of the rock samples of the DIC

A - Ti/Zr diagram of Condie (1989); B - Ba/Nb diagram of D'Orazio et al. (2004); C - Cr vs. Y diagram of Pearce (1982); D - Th(n)/Nb(n) diagram of Saccani (2015); symbols are the same as in Figure 5; abbreviations: AFC - assimilation-fractional crystallization, FC - fractional crystallization, MORB - mid-ocean ridge basalts, OIB-CE - ocean-island type enrichment, PM - primitive mantle, SSZ-E - supra-subduction zone enrichment, VAB - volcanic arc basalts, WPB - within-plate basalts 


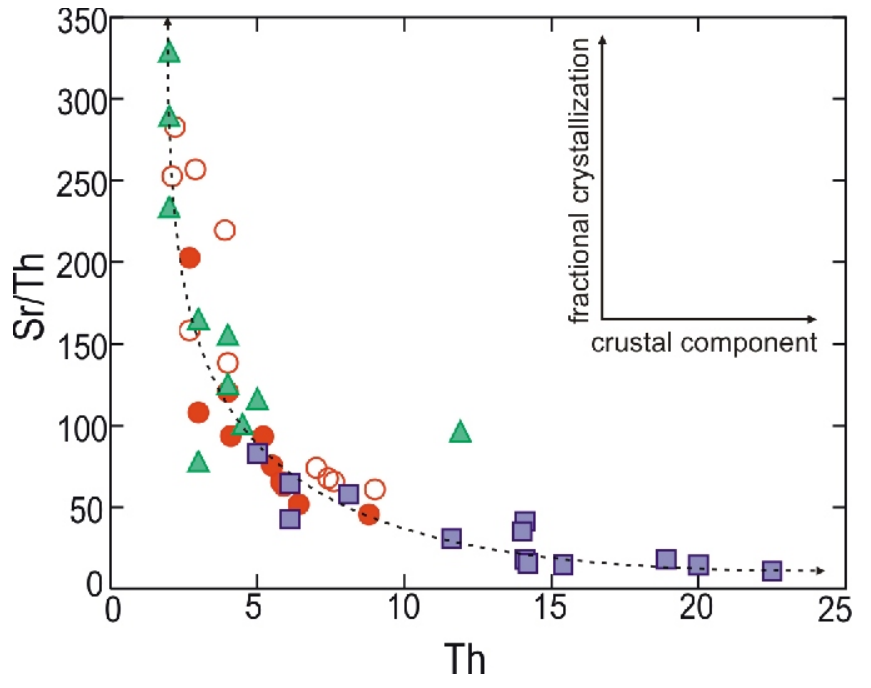

Fig. 9. Sr/Th vs. Th diagram (based on Zou et al., 2008) showing the two magmatic trends for the samples of DIC: fractional crystallization for basic samples and crustal contamination for felsic samples

Symbols are the same as in Figure 5

lavas, pelagic fossil-bearing deposits and also monzodioritegabbro bodies. A similar succession in the neighboring area has been called a "Southern Caspian Sea ophiolite" by Salavati et al. (2013). However, the DIC cannot be taken in consideration as an ophiolitic complex, because it lacks some of the diagnostic features such as sheeted dykes and ultramafic rocks, or these may be hidden below the upper units. However, based on the following three features, it seems that it could have belonged to an embryonic (or rift-related) ocean basin in which effusive eruptions occurred intermittently: (1) the pillow lava unit is a distinctive feature indicating deep submarine volcanic eruptions (e.g., Cas and Wright, 1988); (2) the alternating pelagic deposits (of an open and deep marine environment of Santonian-Maastrichtian age) and the basaltic sheet lavas
(Fig. 3A) indicate intermittent, effusive submarine eruptions in a relatively deep sedimentary basin; (3) the segregation vesicles (Fig. 4B) in the basaltic sheet lavas and also the swallow-tail plagioclase crystals are features that have been proposed by some researchers (e.g., Smith, 1967; Lofgren, 1974; Mevel and Velde, 1976; Swanson and Schiffman, 1979; Anderson et al., 1984; Sanders, 1986; Caroff et al., 2000; Vernon, 2004) as indicating deep submarine eruptions. According to Lofgren (1974), Mevel and Velde (1976) and Swanson and Schiffman (1979), hyalomicrolithic and swallow-tail plagioclase crystals may be produced by a high rate of undercooling of lava at great depths of water. Similarly, the formation of segregation vesicles (Fig. 4B) has been explained as a feature of magma entering an environment with high external pressure (Smith, 1967; Anderson et al., 1984; Sanders, 1986; Caroff et al., 2000; Vernon, 2004) such as great ocean depth (Smith, 1967).

\section{IMPLICATIONS FOR TECTONIC SETTING}

Two lines of evidence may help discover the possible tectonic setting of the DIC: firstly, the occurrence of an embryonic oceanic basin during the Cretaceous based on evidence such as the basaltic pillow lavas, the basaltic sheet lava flows interbedded with pelagic deposits along with monzodioriticgabbroic and felsic (possible equivalents to plagiogranites) bodies which may be considered as an ophiolitic sequence and so could imply a primitive or juvenile oceanic crust; the other line of evidence is the geochemical characteristics of the evolving magmas due to the effects of the tensional within-plate regimes (similar to OIB-type or plume-type magmas) and then compressive regimes (the magmas of supra-subduction zones).

Thus, we consider that the DIC had been formed in a tensional regime of supra-subduction zones far from an arc setting in which an embryonic rift-related ocean was affected by subduction processes. This inferred model (Fig. 11) can be supported by the occurrence of subduction of the Neotethys ocean beneath the central Iran microcontinent in the Late Cretaceous, ophiolite emplacement during the Zagros orogeny and the formation of the Cretaceous volcanic rocks in the similar
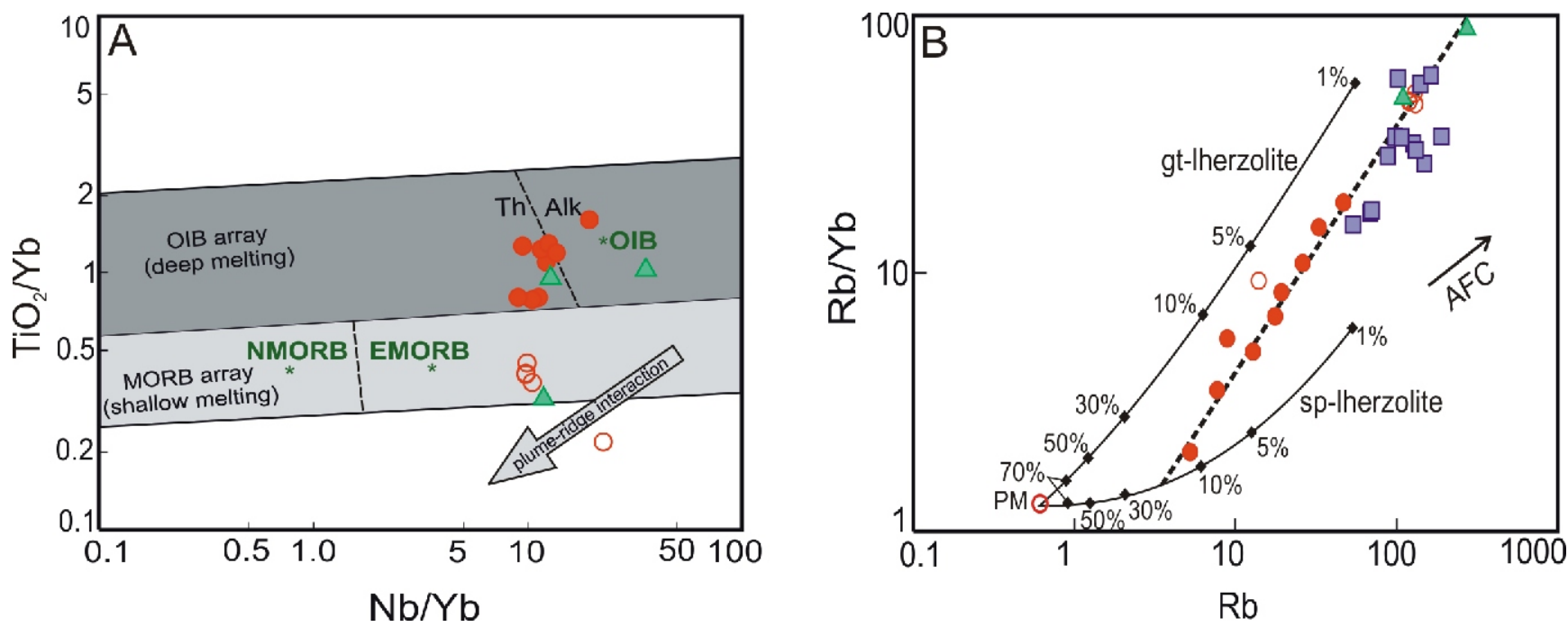

Fig. 10. Petrogenetic diagram of the rock samples from the area studied

A - Yb-normalized diagram of $\mathrm{TiO}_{2}$ vs. $\mathrm{Nb}$ (Pearce, 2008), note the evolving trend of samples between OIB and MORB sources (or plume-ridge interaction); $\mathbf{B}$ - plotted samples on the Rb/Yb vs. Rb diagram of Özdemir et al. (2006), note the AFC trends of the samples from a spinel Iherzolite source; symbols are the same as in Figure 5; Abbreviations: Alk- alkaline, Th- Tholeiite, other as on Figures 7 and 8 

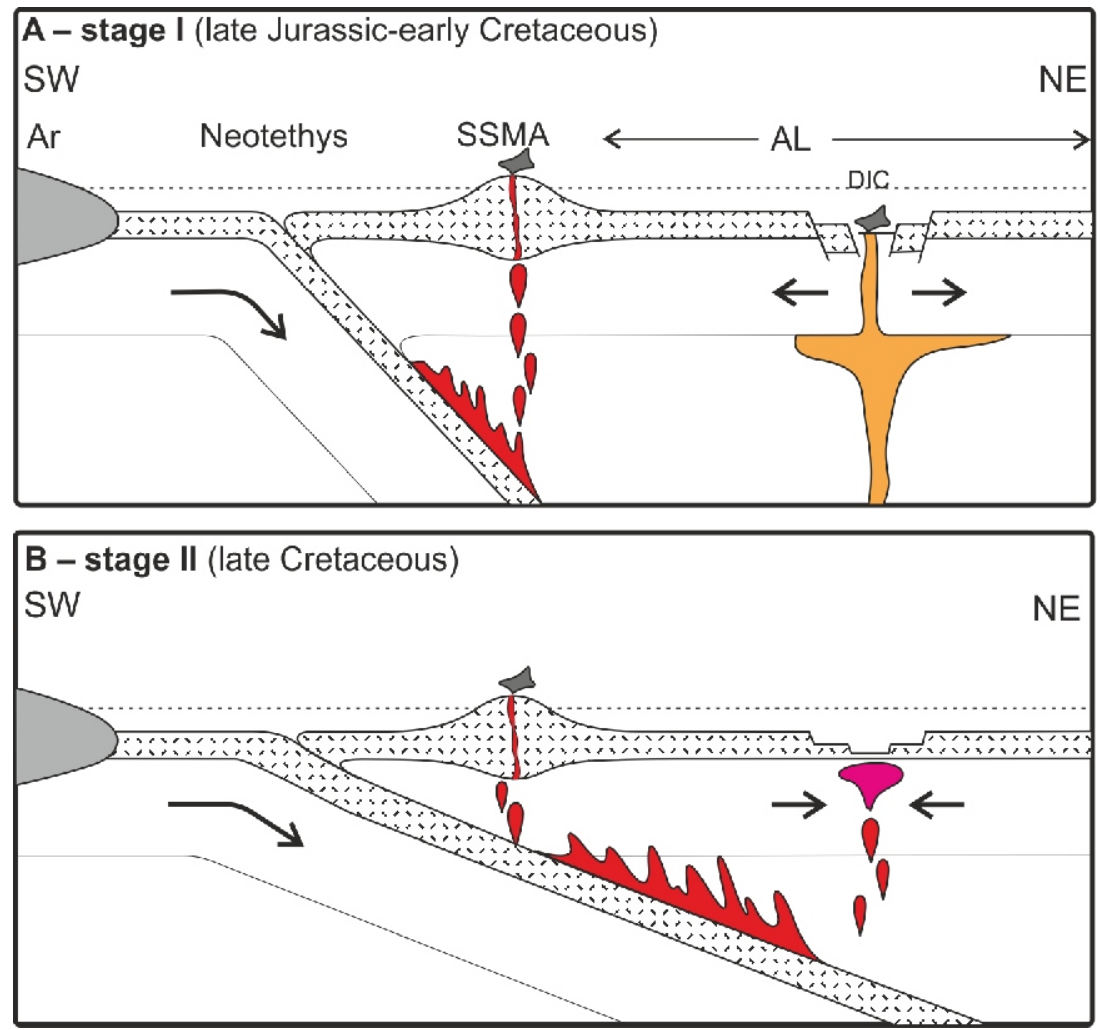

Fig. 11. Schematic illustration of the tectono-magmatic evolution of the DIC in two stages: A - formation of OIBs in a back-arc basin in a tensional regime due to relatively high-angle subduction in the Late Jurassic-Early Creraceous and B - interaction of arc- and OIB-related magmas in a compressive regime due to a flatter subduction in the Late Cretaceous

\section{AL - Alborz; Ar - Arabia; DIC - Deylaman igneous complex; SSMA - Sanandaj-Sirjan magmatic arc}

stratigraphic horizon to the UDMA (e.g., Ghazi et al., 2003; Hassanipak and Ghazi, 2000; Allahyari et al., 2010; Saccani et al., 2013).

Such a hypothesis is consistent with the models presented by some researchers such as Verdel et al. (2011) and Hassanzadeh and Wernicke (2016) who propose that the Mesozoic magmatic arc of SSZ developed in two stages: with steeper subduction in the Late Jurassic-Early Cretaceous followed by the flatter subduction in the Aptian-Albian. In the first stage (i.e., Marianas-type subduction), a back-arc spreading zone was formed and at the second stage (i.e., Chilean-type subduction), the arc magmatism migrated inland. Also, according to Stampfli and Borel (2004), there was a back-arc ocean basin called the "Izanca ocean" with passive margins at 180-121 Ma. Moreover, such tensional back-arc basins have been reported by some authors in different locations of the Zagros orogeny: the "Nain-Baft" basin in the SE (e.g., Shahbpour, 2005; Hosseini et al., 2017) and the "Khoy-Zanjan" basin (e.g., Ajirlu et al., 2016) in the NW.

As a result, it may be suggested that the arc magmatism in the Late Cretaceous was a prelude for the Eocene magmatic flare-up (Verdel et al., 2011) in the UDMA and the Alborz zone.

\section{CONCLUSION}

The tectonic instabilities of Cretaceous time (Late Cimmerian) in the Alborz zone were initiated after the transgression of a shallow-marine, epicontinental/continental shelf environment on the Lower Jurassic foreland deposits during Middle Jurassic time (Alavi 1996). One of the most important features of these activities was the deep submarine magmatism along the northern slopes of the central and western Alborz zone which occurred intermittently with fossil-bearing pelagic deposits and prolonged until the Late Cretaceous (Santonian-Maastrichtian).

Although a deep and open oceanic basin may be inferred from facies and micropalaeontology evidence, the geochemical characteristics of the magmatic rocks imply that they are likely associated with an embryonic, rift-related oceanic basin whose magmas come from a deep and enriched mantle source (possibly spinel Iherzolite in composition) in a supra-subduction zone modified by deep mantle plume components (OIBs). So, a two-stage scenario may be proposed for the Cretaceous magmatism in the Alborz zone associated with the SSZ arc magmatism that is consistent with the models suggested by different researchers (e.g., Verdel et al., 2011; Hassanzadeh and Wernicke, 2016; Hosseini et al., 2017): firstly, the subduction of steeper oceanic lithosphere of the Neotethys in the Late Jurassic-Early Cretaceous and formation of a back-arc basin, followed by flatter subduction and so inland migration of the magmatic arc in the Late Cretaceous. As a result, the younger magmatic events in the Cenozoic (mostly Eocene) took place in the inner parts of the upper plate and a little farther away from the geosuture zone.

Acknowledgements. We would like to thank Dr. A. Dehbozorgi for identifying microfossils and also Mr. S. Sedighi for his fruitful comments to improve the language of this paper. 


\section{REFERENCES}

Agard, P., Omrani, J., Jolivet, L., Whitechurch, H., Vrielynck, B. Spakman, W., Monié, P., Meyer, B., Wortel, R., 2011. Zagros orogeny: a subduction-dominated process. Geological Magazine, 148: 692-725.

Ajirlu, M., Moazzen, M., Hajialioghli, R., 2016. Tectonic evolution of the Zagros Orogen in the realm of the Neotethys between the Central Iran and Arabian Plates: an ophiolite perspective. Central European Geology, 59: 1-27.

Alavi, M., 1996. Tectonostratigraphic synthesis and structural style of the Alborz Mountain system in Northern Iran. Journal of Geodynamics, 21: 1-33.

Allahyari, Kh., Saccani, E., Pourmoafi, M., Beccaluva, L., Masoudi, F., 2010. Petrology of mantle peridotites and intrusive mafic rocks from the Kermanshah ophiolitic complex (Zagros belt, Iran): implications for the geodynamic evolution of the Neo-Tethyan oceanic branch between Arabia and Iran. Ofioliti, 35: 71-90.

Allen, M.B., Blanc, E.J.P., Walker, R., Jackson, J., Talebian, M., Ghassemi, M.R., 2006. Contrasting styles of convergence in the Arabia-Eurasia collision: why escape tectonics does not occur in Iran. Geological Society of America Special Paper, 409 579-589.

Anderson, A.T., Swihart, G.H., Artioli, G., Geiger, C.A., 1984 Segregation vesicles, gas filter-pressing, and igneous differentiation. Journal of Geology, 92: 55-72.

Annells, R.N., Arthurton, R.S., Bazely, R.A., Davis, R.G., 1975. Explanatory text of the Qazvin and Rasht quadrangles map $(1: 250$ 000). Geological Survey of Iran, No. E3, E4.

Baharfiruzi, Kh., Shafeii, A.R., Azhdari, A., Karimi, H.R., 2003 Geological map of Javaherdeh quadrangle map (1:100 000 scale). Geological Survey of Iran.

Berberian, M., King G.C.P., 1981. Towards a paleogeography and tectonic evolution of Iran. Canadian Journal of Earth Sciences, 18: $210-265$.

Boynton, W.V., 1984. Cosmochemistry of the rare earth elements: meteorite studies. In: Rare Earth Element Geochemistry (ed. P. Henderson): 63-114. Amsterdam, Elsevier.

Caroff, M., Maury, R.C., Cotton, J., Clément J.P., 2000. Segregation structures and vapor-differentiated basaltic flows. Bulletin of Volcanology, 62: 87-171.

Cas, R.A.F., Wright, J.V., 1988. Volcanic Successions: Modern and Ancient. Unwin Hyman, London.

Chiu, H.Y., Chung, S.L., Zarrinkoub, M.H., Mohammadi, S.S., Khatib, M.M., lizuka, Y., 2013. Zircon U-Pb age constraints from Iran on the magmatic evolution related to Neotethyan subduction and Zagros orogeny. Lithos, 162-163: 70-87.

Condie, K.C., 1989. Geochemical changes in basalts and andesites across the Archean-Proterozoic boundary: identification and significance. Lithos, 23: 1-18.

D’Orazio, M., Innocenti, F., Manetti, P., Haller, M.J., 2004. Cenozoic back-arc magmatism of the southern extra-Andean Patagonia $\left(44^{\circ} 30^{\prime}-52^{\circ} \mathrm{S}\right)$ : a review of geochemical data and geodynamic interpretations. Revista de la Asociación Geológica Argentina, 59: 525-538.

Doroozi, R., Vaccaro, C., Masoudi, F., Petrini, R., 2016. Cretaceous alkaline volcanism in south Marzanabad, northern central Alborz, Iran: Geochemistry and petrogenesis. Geoscience Frontiers, 7: 937-951.

Ghazi, A.M., Pessagno, E.A., Hassanipak, A.A., Kariminia, S.M., Duncan, R.A., Babaie, H.A. 2003. Biostratigraphic zonation and ${ }^{40} \mathrm{Ar}^{39} \mathrm{Ar}$ ages for the Neotethyan Khoy ophiolite of NW Iran. Palaeogeography, Palaeoclimatology, Palaeoecology, 193: 311-323.

Haghnazar, Sh., 2012. Petrology, geochemistry and tectonic setting of Javaherdasht Cretaceous gabbro in the north part of Alborz Mountains, East of Guilan, north of Iran: a part of ophiolite sequence or intra-continental rift? (in Farsi). Iranian Journal of Petrology, 10: 79-94.
Haghnazar, Sh., Malakotian, S., 2009. Petrography and Geochemistry of the Javaherdasht basalts (east of Guilan Province): the investigation of the role of crystal fractionation and crustal contamination in the magmatic evolution (in Farsi). Iranian Journal of Crystallography and Mineralogy, 17: 253-266.

Haghnazar, Sh., Malakotian, S., Allahyari, Kh., 2015 Tectono-magmatic setting of Cretaceous pillow basalts in the north part of the Alborz Mountains in east of Guilan province (north of Iran): a part of ophiolite sequence or intra-continental rift? (in Farsi). Geosciences, 24: 171-182.

Haghnazar, Sh., Shafeie, Z., Sharghy, Z., 2016. Petrogenesis and tectonic setting of an basalt-trachyte-rhyolite suite in the Spili area (south of Siahkal), north of Iran: evidences of continental rift-related bimodal magmatism in Alborz (in Farsi). Iranian Journal of Petrology, 27: 43-60.

Harker, A. 1909. The Natural History of Igneous Rocks. Methuen \& Co., London.

Hassanipak, A.A., Ghazi, A.M., 2000. Petrology, geochemistry and tectonic setting of the Khoy ophiolite, northwest Iran: implications for Tethyan tectonics. Journal of Asian Earth Sciences, 18 109-121.

Hassanzadeh, J., Wernicke, B.P., 2016. The Neotethyan Sanandaj-Sirjan zone of Iran as an archetype for passive margin-arc transitions Tectonics, 35: 586-621.

Hosseini, M.R., Hassanzadeh, J., Alirezaei, S., Sun, W., Li, C.Y., 2017. Age revision of the Neotethyan arc migration into the southeast Urumieh-Dokhtar belt of Iran: geochemistry and U-Pb zircon geochronology. Lithos, 284-285: 296-309.

Hultberg, S.U., Malmgren, B.A., 1986. Dinoflagellate and planktonic foraminiferal paleobathymetrical indices in the Boreal uppermost Cretaceous. Micropaleontology, 32: 316-323.

Jafari Sough, R., Asiabanha, A., Nasrabadi, M., 2018. Geochemistry of Cretaceous hydromagmatic lava flows in Separdeh district, NE Qazvin, central Alborz (in Farsi). Iranian Journal of Crystallography and Mineralogy, 26: 717-732.

Keller, G., Adatte, T., Stinnesbeck, W., Luciani, V., Karoui-Yaakoub, N., Zaghbib-Turki, D., 2002. Paleoecology of the Cretaceous-Tertiary mass extinction in planktonic foraminifera. Palaeogeography, Palaeoclimatology, Palaeoecology, 178: 257-297.

Lam, P.J., 2002. Geology, geochronology, and thermochronology of the Alam Kuh area, central Alborz Mountains, northern Iran. MSc. thesis, University of California, Los Angeles.

Le Maitre, R.W., Streckeisen, A., Zanettin, B., Le Bas, M.J., Bonin, B., Bateman, P., Bellieni, G., Dudek, A., Efremova, S., Keller, J., Lameyre, J., Sabine, P.A., Schmid, R., Sørensen, H., Wooley, A.R., 2002. Igneous rocks, a classification and glossary of terms. Recommendations of the International Union of Geological Sciences Subcommission on the Systematics of Igneous Rocks, 2nd ed. Cambridge. Cambridge University Press.

Lofgren, G.E., 1974. An experimental study of plagioclase crystal morphology: isothermal crystallization. American Journal of Science, 274: 243-273.

Luciani, V., 2002. High-resolution planktonic foraminifera analysis from the Cretaceous-Tertiary boundary at Ain Settara (Tunisia): evidence of an extended mass extinction. Palaeogeography, Palaeoclimatology, Palaeoecology, 178: 299-319.

Mevel, C., Velde, D., 1976. Clinopyroxenes in Mesozoic pillow lavas from the French Alps: influence of cooling rate on compositional trends. Earth and Planetary Science Letters, 32 : 158-164.

Özdemir, Y., Karaođlu, Ö., Tolluođlu, A.Ü., Gülec, N., 2006. Volcanostratigraphy and petrogenesis of the Nemrut stratovolcano (East Anatolian High Plateau): the most recent post-collisional volcanism in Turkey. Chemical Geology, 226: 189-211. 
Pearce, J.A., 1982. Trace element characteristics of lavas from destructive plate boundaries. In: Andesites (ed. R.S. Thorpe): 525-548. John Wiley \& Sons, New York.

Pearce, J.A., 1983. Role of the sub-continental lithosphere in magma genesis at active continental margin. In: Continental Basalts and Mantle Xenoliths (eds. C.J. Hawkesworth and M.J. Norry): 230-249. Shiva, Nantwich, UK.

Pearce, J.A., 1996. A user's guide to basalt discrimination diagrams. Geological Association of Canada, Short Course Notes, 12: $79-113$.

Pearce, J.A., 2008. Geochemical fingerprinting of oceanic basalts with applications to ophiolite classification and the search for Archean oceanic crust. Lithos, 100: 14-48.

Saccani, E., Allahyari, Kh., Beccaluva, L., Bianchini, G., 2013 Geochemistry and petrology of the Kermanshah ophiolites (Iran): implication for the interaction between passive rifting oceanic accretion, and OIB-type components in the Southern Neo-Tethys Ocean. Gondwana Research, 24: 392-411.

Saccani, E., 2015. A new method of discriminating different types of post-Archean ophiolitic basalts and their tectonic significance using Th-Nb and Ce-Dy-Yb systematics. Geoscience Frontiers, 6: 481-501.

Salavati, M., Kananian, A., Noghreyan, M., 2013. Geochemica characteristics of mafic and ultramafic plutonic rocks in southern Caspian Sea Ophiolite (Eastern Guilan). Arabian Journal of Geoscience, 6: 4851-4858.

Sanders, I.S., 1986. Gas filter-pressing origin for segregation vesicles in dykes. Geological Magazine, 123: 67-72.

Shahabpour, J., 2005. Tectonic evolution of the orogenic belt in the region located between Kerman and Neyriz. Journal of Asian Earth Sciences 24: 405-417.

Shahin, A.M., 1992. Contribution to the foraminiferal biostratigraphy and paleobathymetry of the Late Cretaceous and Early Tertiary in the western Central Sinai, Egypt. Revue de Micropaleontologie, 35: 157-175.

Smith, R.E., 1967. Segregation vesicles in basaltic lava. American Journal of Science, 265: 696-713.

Stampfli, G.M., Borel, G.D., 2004. The TRANSMED transects in space and time: constraints on the Paleotectonic evolution of the Mediterranean Domain. In: The TRANSMED Atlas: The Mediterranean Region from Crust to Mantle (eds. W. Cavazza, F. Roure, W. Spakman, G.M. Stampfli and P. Ziegler): 53-80. Springer Verlag.

Sun, S.S., McDonough, W.F., 1989. Chemical and isotopic systematic of oceanic basalts: implications for mantle composition and processes. Geological Societe Special Publications, 42: 313-345.

Swanson, S.E., Schiffman, P., 1979. Textural evolution and metamorphism of pillow basalts from the Franciscan Complex, Western Matin County, California. Contributions to Mineralogy and Petrology, 69: 291-299.

Taki, S., 2017. The role of fractional crystallization in the evolution of magma of the Upper Cretaceous volcanic and subvolcanic rocks from the Nageleh Sar Syncline, south Mahmood Abad, North Iran (in Farsi). Iranian Journal of Crystallography and Mineralogy, 25: 501-512.

Verdel, C., Wernicke, B.P., Hassanzadeh, J., Guest, B., 2011. A Paleogene extensional arc flare-up in Iran. Tectonics, $\mathbf{3 0}$ TC3008, doi:10.1029/2010TC002809

Vernon, R.H., 2004. A Practical Guide to Rock Microstructure. Cambridge University Press.

Winchester, J.A., Floyd, P.A., 1977. Geochemical discrimination of different magma series and their differentiation products using immobile elements. Chemical Geology, 20: 325-343.

Winter, J.D., 2014. Principles of Igneous and Metamorphic Petrology (2nd ed.). Pearson New Education Limited.

Xia, L., Xu, X., Li, X., Ma, Z., Xia, Z., 2012. Reassessment of petrogenesis of Carboniferous-Early Permian rift-related volcanic rocks in the Chinese Tianshan and its neighboring areas. Geoscience Frontiers, 3: 445-471.

Yadollah-Pour, A., Asiabanha, A., Dehbozorgi A., 2019. Cretaceous subaqueous volcanic alternations in Chalus Formation (Meres section), northern Alborz. 26th Symposium of Crystallography and Mineralogy of Iran (SCMI), Qazvin, Iran.

Zou, H., Fan, Q., Yao, Y., 2008. U-Th systematics of dispersed young volcanoes in NE China: Asthenosphere upwelling caused by piling up and upward thickening of stagnant Pacific slab. Chemical Geology, 255: 134-142. 